\title{
Simple topological measures and a lifting problem
}

by

\author{
Finn F. Knudsen (Trondheim) \\ This paper is dedicated to my colleague Johan Aarnes who has introduced \\ me and many others to tread on this beautiful virgin ground \\ where exotic topological measures live
}

\begin{abstract}
We state a certain lifting conjecture and prove it in the case of a torus. From this result we are able to construct a connected dense subset of the space of intrinsic simple topological measures on the torus, consisting of push forwards of compactly supported generalized point-measures on the universal covering space. Combining this result with an observation of Johansen and Rustad, we conclude that the space of simple topological measures on a torus is connected.
\end{abstract}

\section{Contents}

Introduction

1. The lifting conjecture 203

2. Outline of the proof 206

3. Properties of vh-curves and vh-domains on a torus 207

4. Simple topological measures and vh-fibers 216

5. Special domains $\quad 216$

6. Pseudo-vh ${ }_{\sigma}$-domains and pseudo-roofs 223

7. Moving directed pseudo- $\mathrm{vh}_{\sigma}$-domains 232

$\begin{array}{ll}\text { 8. Reduction } 234 & 232\end{array}$

9. Lifting $\mathrm{vh}_{\sigma}$-fibers right $\quad 237$

10. Density and connectedness 239

$\begin{array}{ll}\text { References } & 240\end{array}$

Introduction. By a space we will mean a topological space that is Hausdorff, connected, locally connected and semi-locally one-connected. Recall that semi-locally one-connected is the property that ensures that the space has a universal covering. Topological measures will only be considered

2000 Mathematics Subject Classification: Primary 28A25; Secondary 28A51.

Key words and phrases: topological measure, quasi-linear functional, vh-curves. 
on compact spaces. Note that if $X$ is a compact space, its universal covering space need not be compact.

Topological measures were first introduced by Johan Aarnes under the name of quasi-measures. Perhaps their proper name should be Aarnes measures. They differ from conventional measures mainly because they need not be subadditive. In fact, the measure of a union of measurable sets, if measurable, might be strictly greater than the sum of the measures of the individual sets. The definition given by Aarnes is essentially the following.

Definition 0.1. A topological measure on a compact space $X$ is a nonnegative function $\mu$, defined on the collection of open and closed subsets of $X, \mathcal{A}(X)$, with the following properties.

(a) For any pairwise disjoint family $\left\{A_{i}\right\}_{i=1}^{n}$ of sets in $\mathcal{A}(X)$ whose union $\bigcup_{i=1}^{n} A_{i}$ also belongs to $\mathcal{A}(X)$, we have

$$
\mu\left(\bigcup_{i=1}^{n} A_{i}\right)=\sum_{i=1}^{n} \mu\left(A_{i}\right) .
$$

(b) For any open set $U, \mu(U)=\sup \{\mu(C) \mid C \subseteq U, C$ closed $\}$.

A topological measure is called normalized if $\mu(X)=1$, and simple if its only values are 0 and 1 . The set of simple topological measures on $X$ is denoted by $X^{*}$.

The reader may verify that a topological measure is an increasing function on $\mathcal{A}(X)$ partially ordered by inclusion. The restrictions of Borel measures to closed or open sets are topological measures. These are exactly the topological measures that are subadditive. The important discovery of Aarnes was the existence of a non-Borel topological measure [1]. With the help of an integration theory for continuous functions, this non-Borel topological measure gave an example of a non-linear quasi-state on a commutative $C^{*}$-algebra.

A subset of a space is called solid if both it and its complement are connected. Intuitively this means the absence of holes. In [9] it was proved that a simple topological measure is completely determined by its restriction to the family of closed solid subsets. Later this was proved by Aarnes for general topological measures [2]. Unless a space $X$ has a certain topological property, namely that the union of a pairwise disjoint family of closed solid sets remain co-connected, it is very hard to verify whether or not a realvalued function on the closed solid sets is the restriction of a topological measure. Among insiders the spaces with this property are called spaces of "genus" zero, or $q$-spaces. This particular genus is defined in [2], and should be called the Aarnes genus. One may use the Mayer-Vietoris sequence to show that the property that disjoint unions of co-connected sets stay 
co-connected holds if the Čech cohomology group $H^{1}(X, \mathbb{Z})$ vanishes. For reasonable spaces the converse is also true (see [9]). On spaces of Aarnes genus zero, one can construct topological measures quite freely, and it is proved in [9] that this construction yields arbitrarily good approximations to any simple topological measure. An amazing result, proved by Butler in [4], is that on $q$-spaces which are $C W$-complexes of dimension at least 2, arbitrary topological measures can be approximated by extreme topological measures. Simple measures are extreme, but there are non-simple extreme measures (see [2]). On a torus and on generalized tori it is not too hard to construct some topological measures (see [6] in the case of general topological measures, and [9] and [10] in the case of simple topological measures). On the torus some simple topological measures kill all contractible sets. These measures are intrinsic and they are simpler to deal with because the space of intrinsic simple topological measures has a product structure and we can deal with one component at a time. There is one component for each maximal rank one subgroup $\sigma$ of the fundamental group of the torus.

1. The lifting conjecture. In this section we state a general conjecture and the main theorem, and we make some reductions. For any family $\mathcal{F}$ of subsets of a space $X$ we consider the $\operatorname{graph} G(\mathcal{F})$ of $\mathcal{F}$. It is the simple graph whose set of nodes is $\mathcal{F}$, and whose set of edges is $\left\{\left\{F, F^{\prime}\right\} \mid F, F^{\prime} \in \mathcal{F}\right.$, $\left.F \neq F^{\prime}, F \cap F^{\prime} \neq \emptyset\right\}$. In other words, two distinct nodes are joined by an edge if they intersect.

Definition 1.1. A family $\mathcal{F}$ of connected subsets of a space $X$ will be called linked if its graph $G(\mathcal{F})$ is connected, and strongly linked if $G(\mathcal{F})$ is complete $\left(^{1}\right)$.

The next definition is a crucial one.

Definition 1.2. Let $p: Y \rightarrow X$ be a covering map and let $\mathcal{F} \subseteq P(X)$ and $\mathcal{G} \subseteq P(Y)$ be strongly linked families of connected subsets of $X$ and of $Y$. We will say that $\mathcal{G}$ lifts $\mathcal{F}$ if there is a unique one-to-one correspondence $\mathcal{F} \stackrel{\prime}{\rightarrow} \mathcal{G}$ such that for each $F \in \mathcal{F}, p$ maps $F^{\prime}$ onto $F$ and the restriction $\left.p\right|_{F^{\prime}}: F^{\prime} \rightarrow F$ is a covering map.

Definition 1.3. Let $p: Y \rightarrow X$ be a covering map and let $\mathcal{F} \subseteq P(X)$ be a strongly linked family of connected subsets of $X$. We will say that $\mathcal{F}$ is liftable to $Y$, or $p$-liftable, if there is a strongly linked family $\mathcal{G}$ of connected subsets of $X$ which lifts $\mathcal{F}$. Note that if $\mathcal{F}$ is liftable to the universal covering

$\left({ }^{1}\right)$ Some authors call strongly linked families simply linked families, but I feel that the term linked only suggests connectedness of the graph. In [9] strongly linked families are called PNI-families, which is short for pairwise non-empty intersection. 
space, then it is liftable to any covering space. We will call such a family universally liftable.

LEMma 1.4. Let $\mathcal{E}$ and $\mathcal{F}$ be strongly linked families of connected subsets of $X$, and suppose every member of $\mathcal{F}$ contains a member of $\mathcal{E}$. Then if $\mathcal{E}$ is p-liftable, so is $\mathcal{F}$.

Definition 1.5. If $\mu$ is a simple topological measure and $\mathcal{F}$ is any family of closed or open subsets of a space $X$, we denote by $\mathcal{F}_{\mu}$ the subset $\{A \in \mathcal{F} \mid \mu(A)=1\}$. A family $\mathcal{F}$ of subsets of $X$ such that $\mu(F)=1$ for each $F \in \mathcal{F}$ will be called a $\mu$-family.

If $X$ is a manifold and $\mathcal{F}$ is a finite universally liftable strongly linked family of connected open or closed subsets of $X$, then $\mathcal{F}$ is a $\mu$-family for a simple topological measure $\mu$ on $X$, and this $\mu$ can be chosen to be the direct image of a generalized point-measure supported by a compact subspace of the universal covering space of $X$ (see [9]). In this article, however, we shall be more concerned with the converse. We believe that the following holds.

Conjecture 1.6. If $\mu$ is a simple topological measure on a space $X$, then any $\mu$-family of connected open or closed subsets of $X$ is universally liftable.

The reason for this conjecture is that we can prove it when $X$ is a torus, and the family consists of non-contractible sets. The techniques we use may possibly work also for the $n$-torus.

In the rest of this paper $X$ is a torus, and $p: Y \rightarrow X$ is a universal covering space for $X$.

Definition 1.7. Let $\left(^{2}\right) \sigma \subseteq \pi_{1}(X)$ be a maximal rank one subgroup of the fundamental group of $X$. A connected open set $U \subseteq X$ will be called a $\sigma$-set of $X$ if the inclusion induces an isomorphism of $\pi_{1}(U)$ onto $\sigma$. A connected closed set will be called a $\sigma$-set of $X$ if its complement has a connected component which is a $\sigma$-set. The set of all open and closed $\sigma$-sets is denoted by $\mathcal{A}_{\sigma}$.

Definition 1.8. Let $\mathbb{P}$ denote the set of all maximal rank one subgroups of $\pi_{1}(X)$. The two-sided sets of $X$ are the open or closed subsets of $X$ that are $\sigma$-sets for some $\sigma \in \mathbb{P}$,

$$
\mathcal{A}_{I I}=\bigcup\left\{\mathcal{A}_{\sigma} \mid \sigma \in \mathbb{P}\right\} .
$$

There is a generalization of two-sided sets to $n$-sided sets (see [6]). It will take the rest of the paper to prove the following theorem.

$\left({ }^{2}\right)$ We drop base-points since $\pi_{1}(X)$ is commutative. 
TheOREM 1.9. For any simple topological measure $\mu$ on the torus, the family $\mathcal{A}_{I I, \mu}$ is universally liftable.

Lemma 1.10. A family $\mathcal{F} \subseteq \mathcal{A}_{I I}$ is liftable if and only if $\mathcal{F} \cap \mathcal{A}_{\sigma}$ is liftable for each $\sigma \in \mathbb{P}$.

Proof. If $\sigma \neq \sigma^{\prime}, F \in \mathcal{A}_{\sigma}$ and $F^{\prime} \in \mathcal{A}_{\sigma^{\prime}}$, and $\widetilde{F}$ and $\widetilde{F^{\prime}}$ are any lifts of $F$ and $F^{\prime}$ to $Y$, then $\widetilde{F} \cap \widetilde{F^{\prime}} \neq \emptyset$.

The group $\sigma$ acts on the left on $Y$. Let $Z$ be the orbit space of this action, and let $r: Y \rightarrow Z$ and $q: Z \rightarrow X$ be the corresponding decomposition of $p: Y \rightarrow X$. Then both $r$ and $q$ are even covers, $Z$ is homeomorphic to a cylinder $\mathbb{R} / \mathbb{Z} \times \mathbb{R}$ and is acted on by $\pi_{1}(X) / \sigma \approx \mathbb{Z}$.

Lemma 1.11. For any $\sigma$-set $A$, the map $r$ induces a one-to-one correspondence between the components of $p^{-1}(A)$ and $q^{-1}(A)$, and the restrictions of $q$ induces homeomorphisms from each connected component of $q^{-1}(A)$ to $A$.

Definition 1.12. For any space $E$, let $\operatorname{Comp}(E)$ be the set of connected components of $E$. If $E$ and $F$ are both subspaces of a common space, we write $\operatorname{Comp}_{F}(E)$ for the set of components meeting $F$.

Lemma 1.13. If $E \subseteq X$ and $F \subseteq X$ be $\sigma$-sets and $E_{0}$ a connected component of $p^{-1}(E)$, then $\operatorname{Comp}_{E_{0}}\left(p^{-1}(F)\right)$ is finite.

Proof. By Lemma 1.11 the map $r$ induces a one-to-one correspondence between $\operatorname{Comp}_{E_{0}}\left(p^{-1}(F)\right)$ and $\operatorname{Comp}_{r\left(E_{0}\right)}\left(q^{-1}(F)\right)$, so it suffices to show that $\operatorname{Comp}_{r\left(E_{0}\right)}\left(q^{-1}(F)\right)$ is finite. Let $\{U, V\}$ be a covering of $X$ by open $\sigma$-sets, and suppose $F \subseteq U$. If $F$ is closed we can choose $U$ to be the complement of a simple closed curve homotopic to a generator of $\sigma$ in $V=$ $X \backslash F$, and if $F$ is open, we choose $U=F$ and $V$ the complement of a simple closed curve homotopic to a generator of $\sigma$ in $U$. Then since $r\left(E_{0}\right)$ is relatively compact, it is covered be a finite number of connected components of $q^{-1}(U)$ and $q^{-1}(V)$.

COROLlary 1.14. A strongly linked family of $\sigma$-sets $\mathcal{F}$ is universally liftable if and only if every finite subfamily $\mathcal{F}^{\prime} \subseteq \mathcal{F}$ is universally liftable.

Proof. The restriction of any lifting of the family $\mathcal{F}$ to any subfamily $\mathcal{F}^{\prime} \subseteq \mathcal{F}$ is a lifting of $\mathcal{F}^{\prime}$ so we only have to look at the "if" part of the corollary. Fix a connected component $E_{0} \in p^{-1}(E)$ for some $E \in \mathcal{F}$ and let

$$
\Gamma=\prod_{F \in \mathcal{F}} \operatorname{Comp}_{E_{0}}\left(p^{-1}(F)\right) .
$$

Let $p_{F}$ denote the projection from $\Gamma$ onto the factor set $\operatorname{Comp}_{E_{0}}\left(p^{-1}(F)\right)$. For any subfamily $\mathcal{F}^{\prime} \subseteq \mathcal{F}$, we define the subset $\Gamma\left(\mathcal{F}^{\prime}\right) \subseteq \Gamma$ of lifts of 
$\mathcal{F}^{\prime} \cup\{E\}$ to be

$$
\Gamma\left(\mathcal{F}^{\prime}\right)=\left\{x \in \Gamma \mid \forall\left(F, G \in \mathcal{F}^{\prime}\right), p_{F}(x) \cap p_{G}(x) \neq \emptyset\right\} .
$$

By definition $\mathcal{F}^{\prime} \cup\{E\}$ is universally liftable if and only if $\Gamma\left(\mathcal{F}^{\prime}\right) \neq \emptyset$. If we give each factor set $\operatorname{Comp}_{E_{0}}\left(p^{-1}(F)\right)$ the discrete topology and $\Gamma$ the product topology, the sets $\Gamma\left(\mathcal{F}^{\prime}\right)$ are closed for finite families $\mathcal{F}^{\prime}$. If $P^{\prime}(\mathcal{F})$ denotes the set of finite subfamilies of $\mathcal{F}$, it follows from Tikhonov's theorem and our assumption that $\bigcap_{\mathcal{F}^{\prime} \in P^{\prime}(\mathcal{F})} \Gamma\left(\mathcal{F}^{\prime}\right) \neq \emptyset$, and this is just another way of saying that the family $\mathcal{F}$ is universally liftable.

DEFINITION 1.15. A neighboring family of a family $\mathcal{F}$ is a family of connected open sets $\mathcal{V}$ together with a one-to-one correspondence $i_{\mathcal{V}}: \mathcal{F} \rightarrow \mathcal{V}$ such that $F \subseteq i_{\mathcal{V}}(F)$ for each $F \in \mathcal{F}$.

LEMma 1.16. If $\mathcal{F}$ is a finite family of $\sigma$-sets, then $\mathcal{F}$ is universally liftable if and only if every neighboring $\sigma$-family is universally liftable.

Proof. By Lemma 1.11, $q$-liftability is equivalent to $p$-liftability, so we consider only $q$-liftability. Pick a connected component $E$ of the pre-image of a member $F \in \mathcal{F}$, and let $\mathcal{F}^{\prime}=\bigcup_{F \in \mathcal{F}} \operatorname{Comp}_{E}\left(q^{-1}(F)\right)$ be the family of connected components of pre-images of members of $\mathcal{F}$ meeting $E$. By Lemma $1.13, \mathcal{F}^{\prime}$ is finite and any lifting of $\mathcal{F}$ can be translated to a lifting $s: \mathcal{F} \rightarrow \mathcal{F}^{\prime}$. For any neighboring $\sigma$-family $\mathcal{V}$ of $\mathcal{F}$, let $\mathcal{V}^{\prime}$ be the neighboring family of $\mathcal{F}^{\prime}$ consisting of the connected components of the pre-images of the members of $\mathcal{V}$, containing members of $\mathcal{F}^{\prime}$. If $E_{1}$ and $E_{2}$ are disjoint members of $\mathcal{F}^{\prime}$, it might happen that $i_{\mathcal{V}^{\prime}}\left(E_{1}\right) \cap i_{\mathcal{V}^{\prime}}\left(E_{2}\right) \neq \emptyset$, but for a given disjoint pair $E_{1}$ and $E_{2}$ of members of $\mathcal{F}^{\prime}$ we can find a neighboring $\sigma$-family $\mathcal{V}$ of $\mathcal{F}$ such that $i_{\mathcal{V}^{\prime}}\left(E_{1}\right) \cap i_{\mathcal{V}^{\prime}}\left(E_{2}\right)=\emptyset$. For each disjoint pair $\left(E_{1}, E_{2}\right)$, we pick such a family and call it $\mathcal{V}_{\left(E_{1}, E_{2}\right)}$. For $F \in \mathcal{F}$, let $i_{\mathcal{V}_{0}}(F)=\bigcap i_{\mathcal{V}_{\left(E_{1}, E_{2}\right)}}(F)$, where the intersection runs over all disjoint pairs. Since $\mathcal{F}^{\prime}$ is finite, $\mathcal{V}_{0}=$ $\left\{i_{\mathcal{V}_{0}}(F) \mid F \in \mathcal{F}\right\}$ is a neighboring $\sigma$-family of $\mathcal{F}$, and $s: \mathcal{F} \rightarrow \mathcal{F}^{\prime}$ is a lift of $\mathcal{F}$ if and only if the composition $s_{0}=i_{\mathcal{V}_{0}} \circ s \circ\left(i_{\mathcal{V}_{0}}\right)^{-1}$ is a lift of $\mathcal{V}_{0}$.

COROllary 1.17. For a simple topological measure $\mu$ on the torus $X$, if for all maximal rank one subgroups $\sigma$ of the fundamental group the family $\mathcal{U}_{\sigma, \mu}$ of open $\mu$-sets is liftable, so is $\mathcal{A}_{I I, \mu}$.

COROLlary 1.18. For a simple topological measure $\mu$ on the torus $X$, the family of all open or closed non-contractible $\mu$-sets is liftable.

2. Outline of the proof. Since we prove only one theorem in this paper, and the proof is rather long and technical, this outline of the proof may be of some help to the reader. Many of the concepts we mention in this section are not yet defined, but we will refer to the appropriate definitions. The reason for basing the proof on $v h_{\sigma}$-curves or fibers (see Sections 3 
and 4$)$ is twofold. First, because the $\sigma$-component $\mu_{\sigma}$ of a simple topological measure $\mu$ (see Section 4) is determined by its restriction to $\mathrm{vh}_{\sigma}$-fibers, and secondly, because the order-function allows us to use induction. The main technical lemma is Key Lemma 8.6, which we use to show that a $\mu$-fiber can be continuously shrunk through $\mu$-fibers to a $\mu$-fiber of lower order. The reader is advised to familiarize herself/himself with this lemma in the particular cases of fibers of order 4, 6 and 8. From the homotopy lifting property of covering maps, continuous shrinkings can be lifted to any covering of the torus, therefore we can shrink any lifting of a $\mu$-fiber to the lifting of a $\mu$-fiber of order 0 . The proof then consists of two rather technical lemmas. The first is Lemma 9.4, which together with induction shows that when a lifted $\mu$-fiber is shrunk to a lifted $\mu$-fiber of order 0 , this fiber is unique. The second is Lemma 9.8, which shows that two distinct lifted $\mu$-fibers that may be shrunk to the same order 0 fiber, intersect.

3. Properties of vh-curves and vh-domains on a torus. As in the previous section, let $p: Y \rightarrow X$ be a universal covering of a torus $X$. We fix a maximal rank one subgroup $\sigma \subseteq \pi_{1}(X)$ and two simple loops $\sigma_{1}$ and $\sigma_{2}$ in $X$ such that the homotopy class $\left[\sigma_{1}\right]$ generates $\sigma$, and the homotopy classes $\left[\sigma_{1}\right]$ and $\left[\sigma_{2}\right]$ generate $\pi_{1}(X)$. In order to talk about vh-curves and vh-domains we need special coordinates on $Y$.

Definition 3.1. A homeomorphism $\mathbf{x}=\left(x_{1}, x_{2}\right): Y \rightarrow \mathbb{R}^{2}$ will be called $\left(\sigma_{1}, \sigma_{2}\right)$-coordinates if the action of the class $\left[\sigma_{i}\right]$ on $Y$ is given by

$$
\mathbf{x}\left(\left[\sigma_{1}\right](y)\right)=\mathbf{x}(y)+(1,0), \quad \mathbf{x}\left(\left[\sigma_{2}\right](y)\right)=\mathbf{x}(y)+(0,1) .
$$

We define $\widetilde{\mathbf{x}}=\left(\widetilde{x}_{1}, \widetilde{x}_{2}\right): X \rightarrow \mathbb{R}^{2} / \mathbb{Z}^{2}$ by the equation $\widetilde{\mathbf{x}} \circ p=(\bmod 1) \circ \mathbf{x}$.

In the following we will fix $\left(\sigma_{1}, \sigma_{2}\right)$-coordinates $\mathbf{x}=\left(x_{1}, x_{2}\right): Y \rightarrow \mathbb{R}^{2}$, and $\left(\widetilde{x}_{1}, \widetilde{x}_{2}\right): X \rightarrow \mathbb{R}^{2} / \mathbb{Z}^{2}$. Using these coordinates, we can define the direction and the turning-direction of consecutive line-segments.

Definition 3.2. If $e$ is any directed line-segment, we write $\mathbf{v}(e) \in \mathbb{R}^{2}$ for its direction unit-vector. If $e^{\prime}$ is a directed line-segment immediately following $e$ in a different, but not opposite direction, we write $\operatorname{dir}\left(e, e^{\prime}\right)$ for the turning-direction, which is either to the right or to the left. We have

$$
\operatorname{dir}\left(e, e^{\prime}\right)= \begin{cases}l & \text { if } \operatorname{det}\left(\mathbf{v}(e), \mathbf{v}\left(e^{\prime}\right)\right)>0 \\ r & \text { if } \operatorname{det}\left(\mathbf{v}(e), \mathbf{v}\left(e^{\prime}\right)\right)<0 .\end{cases}
$$

Definition 3.3. A simple closed curve $\gamma:[a, b] \rightarrow X$ will be called a vh-curve of order $n>0$ with respect to $\mathbf{x}$ if there is a partition $a=t_{0}<t_{1}<$ $\cdots<t_{n}=b$ such that on each interval $\left[t_{i}, t_{i+1}\right]$, we have $\widetilde{\mathbf{x}}(\gamma(t))=\widetilde{\mathbf{x}}\left(\gamma\left(t_{i}\right)\right)+$ $\left(t-t_{i}\right) \mathbf{v}\left(\gamma\left(t_{i}\right)\right)$, where $\mathbf{v}\left(\gamma\left(t_{i}\right)\right) \in\{(1,0),(0,1),(-1,0),(0,-1)\}$ is the velocity of $\gamma$ on the interval $\left[t_{i}, t_{i+1}\right]$, and any two consecutive velocities are linearly independent. This means that the curve turns at the parameter values $t=t_{i}$. 
We write $\operatorname{dir}_{i}(\gamma)$ for the turning-direction at the turning-point $\gamma\left(t_{i}\right)$. We denote by $\operatorname{Turn}(\gamma)=\operatorname{Turn}_{l}(\gamma) \cup \operatorname{Turn}_{r}(\gamma)$ the set of turning-points of $\gamma$. The $i$ th leg of a vh-curve $\gamma$ is the set $\operatorname{leg}_{i}(\gamma)=\left\{\gamma(t) \mid t \in\left[t_{i-1}, t_{i}\right]\right\}$. The length of the $i$ th leg is $\left|\operatorname{leg}_{i}(\gamma)\right|=t_{i}-t_{i-1}$. We denote by $\operatorname{Leg}(\gamma)$ the set of legs of $\gamma$. We will write $|\gamma|$ for the image of $\gamma$, so that $|\gamma|=\bigcup \operatorname{Leg}(\gamma)$. Since the curve bites itself on the tail, we extend the indexing of the turning-points to all of $\mathbb{Z}$ by periodicity. A vh-curve of order 0 is of the form $\widetilde{\mathbf{x}}(\gamma(t))=$ $\widetilde{\mathbf{x}}(\gamma(a))+(t-a) \mathbf{v}(\gamma(a))$ for $0 \leq t \leq 1$.

Definition 3.4. A vh-domain is a domain $D$ bounded by the images of vh-curves. We will say that a vh-domain $D$ is proper if the interior of its complement is also a vh-domain. Note that a proper vh-domain is solid. If $D$ is proper every connected component of the boundary gets an orientation. By choosing a turning-point on the boundary, we get a unique parameterization of that particular connected component of $\partial D$ such that $D$ lies to the left. There are at most two connected components of $\partial D$, and $D$ is contractible if and only if the boundary consists of just one connected component.

Definition 3.5. The shape of a vh-curve $\gamma$ is the string shape $(\gamma)=$ $\operatorname{dir}_{0}(\gamma) \operatorname{dir}_{1}(\gamma) \ldots \operatorname{dir}_{n-1}(\gamma)$. Curves of order 0 have no turns and therefore the shape of such curves is the empty string $\lambda$.

Definition 3.6. Let $v$ and $w$ be strings over the alphabet $\{l, r\}$. A circular occurrence of $v$ in $w$ is a triple $(y, x, z)$ of strings with $x \neq w, y$ and $z$ possibly empty, such that $w=x y$, and $y=v z$ or $v=y z$ and $x=z z^{\prime}$. We have

$\operatorname{circocc}(v, w)=\left\{(y, x, z) \mid x \neq w \wedge w=x y \wedge\left(y=v z \vee\left(v=y z \wedge \exists z^{\prime} x=z z^{\prime}\right)\right)\right\}$.

Intuitively, a circular occurrence of $v$ in $w$ is an occurrence of $v$ in $w$ when we consider $w$ as a circular string. For the number of circular occurrences we write $\operatorname{co}(v, w)=|\operatorname{circocc}(v, w)|$.

Definition 3.7. Strings $v, w$ in $\{l, r\}^{*}$ will be called circularly equivalent, written $v \operatorname{circ} w$, if there are strings $x$ and $y$ such that $v=x y$ and $w=y x$. We let $[w]$ denote the equivalence class of $w$. If we look at $v$ and $w$ as circular strings, $v \operatorname{circ} w$ means that $w$ is obtained from $v$ by a rotation.

The number of circular occurrences of a string $v$ in a string $w$ only depends on the circular equivalence class of $w$, so we can make the following definition.

Definition 3.8. For a string $v$ and a class $[w]$, we let $\operatorname{co}(v,[w])=$ $\operatorname{co}(v, w)$.

EXAMPLE 3.9. $\operatorname{co}(r r, r r)=2$.

Definition 3.10. A turning-point $q=\gamma\left(t_{i}\right)$ of a vh-curve $\gamma$ will be called stair-like if the sequence $\operatorname{dir}_{i-1}(\gamma) \operatorname{dir}_{i}(\gamma) \operatorname{dir}_{i+1}(\gamma)$ is of the form $r l r$ 
or $l r l$. In the first case it is a left stair-like turning-point and in the second case it is a right stair-like turning-point. We denote by $\operatorname{Stair}(\gamma)$ the set of stair-like turning-points of $\gamma$. We have $\operatorname{Stair}(\gamma)=\operatorname{Stair}_{l}(\gamma) \cup \operatorname{Stair}_{r}(\gamma)$.

Observe the following formulas.

Lemma 3.11. For any vh-curve $\gamma$ we have

$\left|\operatorname{Stair}_{l}(\gamma)\right|=|\operatorname{co}(r l r, \operatorname{shape}(\gamma))|, \quad\left|\operatorname{Stair}_{r}(\gamma)\right|=|\operatorname{co}(l r l, \operatorname{shape}(\gamma))|$.

Definition 3.12. We will call a pair $d=\left(d_{1}, d_{2}\right)$ of consecutive turningpoints of a vh-curve $\gamma$ a double-turn if the string $\operatorname{dir}_{\gamma}\left(d_{1}\right) \operatorname{dir}_{\gamma}\left(d_{2}\right)$ is of the form $l l$ or $r r$. In the first case it is a left double-turn and in the second case it is a right double-turn. We denote by $\operatorname{Double}(\gamma)$ the set of double-turns of $\gamma$. We have Double $(\gamma)=\operatorname{Double}_{l}(\gamma) \cup \operatorname{Double}_{r}(\gamma)$.

Definition 3.13. If $d \in \operatorname{Double}(\gamma), \operatorname{mid}_{\gamma}(d)$ will denote the leg between $d_{1}$ and $d_{2}$.

Lemma 3.14. For any vh-curve $\gamma$ we have

$\left|\operatorname{Double}_{l}(\gamma)\right|=\mid \operatorname{co}(l l$, shape $(\gamma))|, \quad| \operatorname{Double}_{r}(\gamma)|=| \operatorname{co}(r r, \operatorname{shape}(\gamma)) \mid$.

Definition 3.15. Let $[w] \in\{r, l\}^{*} /$ circ be a circular class. We choose a representative string $w$, and if $w$ contains both symbols we choose it such that it begins and ends with different symbols. Then $w=a_{1}^{\alpha_{1}} \ldots a_{n}^{\alpha_{n}}$ with $a_{i} \in\{r, l\}, a_{i} \neq a_{i+1}, a_{n} \neq a_{1}$ and $\alpha_{i} \geq 1$. Let $\sigma:\{1, k\} \rightarrow\{1, n\}$ be the increasing function onto the set of indices $i$ such that $\alpha_{\sigma(i)} \geq 2$. Then put $\operatorname{ds}([w])=\left[a_{\sigma(1)} \ldots a_{\sigma(k)}\right]$. We call $\operatorname{ds}([w])$ the derived shape-class of $[w]$ or of $w$.

The reader can check that the derived shape-class is well defined. The next two lemmas are crucial for showing the existence of what we will call order-reducing roofs (see Definition 3.38). If as in Definition 3.15, $w=$ $a_{1}^{\alpha_{1}} \ldots a_{n}^{\alpha_{n}}$ and $a_{\sigma(j)} a_{\sigma(j+1)}$ is a circular occurrence of $r r$ in $\mathrm{ds}([w])$, then on the interval $[\sigma(j), \ldots, \sigma(j+1)], a$ takes alternating values starting and ending with $r$. Similarly a circular occurrence of $l l$ in $\mathrm{ds}([w])$ gives rise to a circular occurrence of $l r l r l \ldots r l$ in $w$. Hence we have proved the formula below.

Lemma 3.16. For any string $w \in\{l, r\}^{*}$ we have the formula

$$
\operatorname{co}(l r l,[w])-\operatorname{co}(r l r,[w])=\operatorname{co}(l l, \mathrm{ds}([w]))-\operatorname{co}(r r, \mathrm{ds}([w])) .
$$

Lemma 3.17. For any string $w$ in $\{r, l\}^{*}$ of length $|w| \neq 1$, we have the equality

$$
\operatorname{co}(r r, w)-\operatorname{co}(l l, w)=\operatorname{co}(r, w)-\operatorname{co}(l, w) .
$$

Proof. We let $F(w)=\operatorname{co}(r r, w)-\operatorname{co}(l l, w)-\operatorname{co}(r, w)+\operatorname{co}(l, w)$, and consider the language $L=\{w \mid F(w)=0\}$. The reader may check that $L$ contains all strings of length 2. By structural induction it suffices to show 
that $L$ is closed under the operations $w \mapsto r w$ and $w \mapsto l w$, and by symmetry we need only consider $w \mapsto r w$. If $w \in L$ and $|w| \geq 2$, there are four distinct cases, $w=l x l, w=l x r, w=r x l$ and $w=r x r$. In the first case $\operatorname{co}(r r, r w)=$ $\operatorname{co}(r r, w)$ and $\operatorname{co}(l l, r w)=\operatorname{co}(l l, w)-1$. In the other three cases $\operatorname{co}(r r, r w)=$ $\operatorname{co}(r r, w)+1$ and $\operatorname{co}(l l, r w)=\operatorname{co}(l l, w)$. This shows that $r w \in L$.

Definition 3.18. For a turning-point $p=\gamma\left(t_{i}\right)$ of a vh-curve $\gamma$ we will denote by $\operatorname{shadow}_{\gamma}(p)$ or $\operatorname{shadow}_{i}(\gamma)$ the open rectangle determined by the two adjacent $\operatorname{legs} \operatorname{leg}_{i}(\gamma)$ and $\operatorname{leg}_{i+1}(\gamma)$.

Definition 3.19. For a double-turn $d=\left(d_{1}, d_{2}\right) \in \operatorname{Double}(\gamma)$, the shadow of $d$ is the intersection $\operatorname{shadow}_{\gamma}(d)=\operatorname{shadow}_{\gamma}\left(d_{1}\right) \cap \operatorname{shadow}_{\gamma}\left(d_{2}\right)$. Shadows are shown in Figure 1.
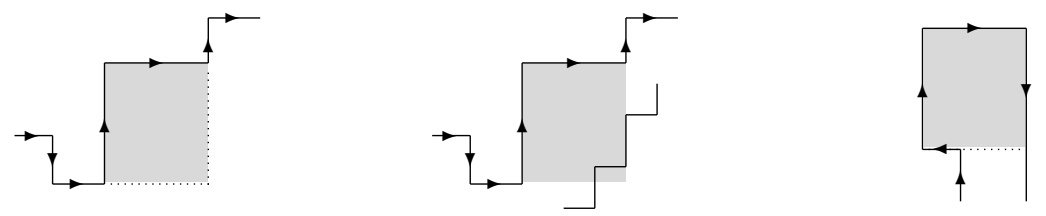

Fig. 1. Left: the shadow of a right stair-like turn. Middle: two primary obstructions. Right: the shadow of a right double-turn.

Definition 3.20. Let $\Gamma$ be a family of vh-curves. A turning-point $p=$ $\gamma\left(t_{i}\right)$ of a curve $\gamma \in \Gamma$ is called $\Gamma$-unobstructed if the intersection of the closure of the shadow of $\gamma\left(t_{i}\right)$ with the union of all the curves in $\Gamma$ is contained in $\operatorname{leg}_{i-1}(\gamma) \cup \operatorname{leg}_{i}(\gamma)$ (see Figure 1). A double-turn $d=\left(\gamma\left(t_{i-1}\right), \gamma\left(t_{i}\right)\right)$ of a curve $\gamma \in \Gamma$ is called $\Gamma$-unobstructed if the intersection of the closure of the shadow of $d$ with the union of all the curves in $\Gamma$ is contained in the union of the five segments $\operatorname{leg}_{i-2}(\gamma), \operatorname{leg}_{i-1}(\gamma), \ldots, \operatorname{leg}_{i+2}(\gamma)$ (see Figure 2).

Definition 3.21. Let $\Gamma$ be a finite disjoint family of simple closed vhcurves. A turning-point $q=\gamma^{\prime}\left(t_{j}\right)$ primary-obstructs a turning-point $p=$ $\gamma\left(t_{i}\right)$ if it lies in the $\gamma$-shadow of $p$, and the intersection of the closed rectangle determined by the points $p$ and $q$ with all of the curves of $\Gamma$ is contained in the union of just the two adjacent legs at $p$ and the point $q$. In symbols, $\operatorname{rectangle}(p, q) \subseteq \operatorname{shadow}_{\gamma}(p)$, and $\overline{\operatorname{rectangle}(p, q)} \cap \bigcup \Gamma \subseteq\{q\} \cup \operatorname{leg}_{i}(\gamma) \cup$ $\operatorname{leg}_{i+1}(\gamma)$.

We leave the following observation to the reader.

LEMMA 3.22. Whenever a turning-point q primary-obstructs a stair-like turn $p, p$ is unique, and therefore the relation of primary obstruction is a partially defined function on the stair-like turns of the family $\Gamma$.

Definition 3.23. Let $\Gamma$ be a finite disjoint family of simple closed vh-curves, and let $d$ and $e$ be double-turns of curves in $\Gamma$. If $\operatorname{mid}(d) \subseteq$ $\overline{\operatorname{shadow}(e)}$, we let shadow $(e, d)$ be the open rectangle determined by the 
shadow of $e$ and the extension of the leg $\operatorname{mid}(d)$. We will say that $d$ primaryobstructs $e$ if $\operatorname{shadow}(e, d) \cap \Gamma=\emptyset$.

DeFinition 3.24. Whenever a double-turn $d$ primary-obstructs $e, e$ is unique and the double-turn $d$ is strictly narrower than the double-turn $e$. Therefore the relation of primary obstruction defines for every such family $\Gamma$ a directed forest which we denote by Forest $(\Gamma)$. The leaves in the forest are the unobstructed double-turns.

Certain double-turns will be called tags. These are the double-turns whose closest successor or predecessor is a turn in the opposite direction. If they are equally close, they must both be opposite turns. We call such tags symmetric. This is the formal definition:

Definition 3.25. A double-turn $d=\left(\gamma\left(t_{i-1}\right), \gamma\left(t_{i}\right)\right)$ will be called a tag if the following holds:

(i) If $\left|\operatorname{leg}_{i-1}(\gamma)\right|<\left|\operatorname{leg}_{i+1}(\gamma)\right|$, then $\operatorname{dir}_{i-2}(\gamma) \neq \operatorname{dir}_{i-1}(\gamma)$.

(ii) If $\left|\operatorname{leg}_{i-1}(\gamma)\right|>\left|\operatorname{leg}_{i+1}(\gamma)\right|$, then $\operatorname{dir}_{i}(\gamma) \neq \operatorname{dir}_{i+1}(\gamma)$.

(iii) If $\left|\operatorname{leg}_{i-1}(\gamma)\right|=\left|\operatorname{leg}_{i+1}(\gamma)\right|$, then $\operatorname{dir}_{i-2}(\gamma) \neq \operatorname{dir}_{i-1}(\gamma)$ and $\operatorname{dir}_{i}(\gamma) \neq$ $\operatorname{dir}_{i+1}(\gamma)$.

Tags are shown in Figure 2.
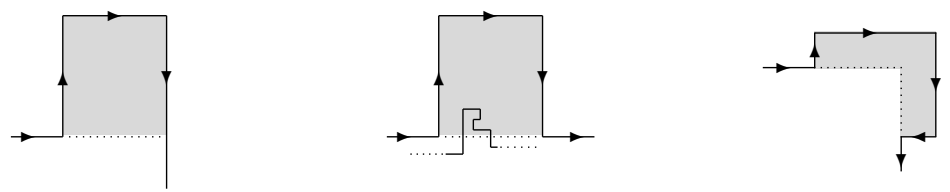

Fig. 2. Left: an unobstructed right tag. Middle: a symmetric tag with one primary obstruction. Right: two consecutive unobstructed right tags or twin tags.

Lemma 3.26. Let $\gamma$ be a vh-curve in a finite disjoint family $\Gamma$ of vhcurves. Let $D=\left\{d_{1}, \ldots, d_{k}\right\}$ be $k$ consecutive double-turns. Let $D^{\prime} \subseteq D$ be the unobstructed ones. Then $D^{\prime} \subseteq\left\{d_{1}, d_{2}, d_{k-1}, d_{k}\right\}$. If $D^{\prime} \neq \emptyset$, we have $D^{\prime} \cap\left\{d_{1}, d_{k}\right\} \neq \emptyset$, and $D^{\prime} \cap\left\{d_{1}, d_{k}\right\}$ are the tags of $D^{\prime}$.

Proof. We encourage the reader to draw the appropriate "fat" spirals. Fatness is because the loose end has to retrace the spiral.

Corollary 3.27. A vh-curve $\gamma$ in a disjoint family $\Gamma$ of vh-curves cannot have three consecutive tags. However, "twin tags" may occur and any subset of them may be $\Gamma$-unobstructed. Unobstructed twin tags look like framed corners (see Figure 2).

LEMMA 3.28. If $\gamma$ is a vh-curve in a disjoint family $\Gamma$ of vh-curves, and if $\gamma$ has a $\Gamma$-unobstructed double-turn, then $\gamma$ has a $\Gamma$-unobstructed tag turning the same way. 
Proof. The proofs for right and left turns are similar, so we may assume that $d=\left(\gamma\left(t_{i-1}\right), \gamma\left(t_{i}\right)\right)$ is an unobstructed left double-turn and not a tag. Assume that $\left|\operatorname{leg}_{i-1}(\gamma)\right| \geq\left|\operatorname{leg}_{i+1}(\gamma)\right|$. Then since $d$ is unobstructed, $\operatorname{dir}_{i+2}(\gamma)=r$, which shows that $\left(\gamma\left(t_{i}\right), \gamma\left(t_{i+1}\right)\right)$ is an unobstructed tag. If $\left|\operatorname{leg}_{i-1}(\gamma)\right| \leq\left|\operatorname{leg}_{i+1}(\gamma)\right|$, it follows for the same reason that $\left(\gamma\left(t_{i-2}\right), \gamma\left(t_{i-1}\right)\right)$ is an unobstructed tag.

Definition 3.29. A simple closed vh-curve of homology class 0 divides the torus into two regions, exactly one of which is contractible. If the curve is the oriented boundary of the contractible region, we will say that the curve is left-turning, and in this case the contractible region lies to the left of the curve. Otherwise the curve is right-turning.

For the most part we shall be concerned with simple closed vh-curves whose free homotopy class generates the subgroup $\sigma$ generated by the free homotopy class of the loop $\sigma_{1}$. Their dependence on the other generator $\sigma_{2}$ is of minor importance.

Definition 3.30. A vh-curve or a vh-domain which is also a $\sigma$-set will be called a $v h_{\sigma}$-curve or a $v h_{\sigma}$-domain. A $v h_{\sigma}$-curve $\gamma$, if properly oriented, is freely homotopic to $\sigma_{1}$. A $\mathrm{vh}_{\sigma}$-domain $D$ which is bounded by a $\mathrm{vh}_{\sigma}$-curve $\gamma^{\prime}$ on the left and a $v_{\sigma}$-curve $\gamma$ on the right will be denoted by $D\left(\gamma, \gamma^{\prime}\right)$. It is proper if and only if the curves are disjoint.

The reason for the right to left ordering is because we have chosen coordinates in such a way that if we stand on the outside of the torus and look in the $x$-direction, which is the direction of $\sigma_{1}$, the $y$-direction is from right to left.

Lemma 3.31. Any simple closed left-turning vh-curve has at least four unobstructed left double-turns. The corresponding statement holds for rightturning curves.

Proof. Let $\gamma$ be a simple left-turning vh-curve. Since the contractible domain defined by $\gamma$ always lies to the left of $\gamma$, no left double-turn can primary-obstruct a left double-turn. Primary obstruction defines a partial function from the right double-turns to the left double-turns. By Lemma 3.17 there are at least four left double-turns that are leaves in the forest of $\gamma$, and we can find two that are not consecutive.

Lemma 3.32. Any simple closed vh ${ }_{\sigma}$-curve of order at least four has at least one unobstructed double-turn in each direction.

Proof. If all turning-points are stair-like, the curve cannot have the right homotopy type, hence the forest of $\gamma$ has leaves, and we may assume that $\gamma$ has an unobstructed right double-turn. Also by the forest property of the primary obstruction relation and Lemma 3.17, not all left double-turns 
are obstructed by left double-turns. Hence either we have an unobstructed left double-turn, or there is a right double-turn $d$ primary-obstructing a left double-turn $e$. In the second case we can choose points $p$ and $q$ in the interior of the legs $\operatorname{mid}(d)$ and $\operatorname{mid}(e)$ so that the line-segment joining $p$ and $q$ in the shadow $e$ is perpendicular to $\operatorname{mid}(d)$ and has length $l$. Let $\delta:[0, l] \rightarrow X$ be the function parameterizing this line-segment, with constant speed, and such that $\delta(0)=p$ and $\delta(l)=q$. Assume that $\gamma:[a, b] \rightarrow X$ has turningpoints $\left\{\gamma\left(t_{0}\right), \gamma\left(t_{1}\right), \ldots, \gamma\left(t_{n}\right)\right\}$, where $a=t_{0}<t_{1}<\cdots<t_{n}=b, p=\gamma(u)$, $q=\gamma(v), t_{0}<u<t_{1}$ and $t_{k}<v<t_{k+1}$. Let $\bar{\gamma}$ denote the periodic extension of $\gamma$. We define two simple closed vh-curves $\gamma^{\prime}:[v-l, b-a+u] \rightarrow X$ and $\gamma^{\prime \prime}:[u, v+l] \rightarrow X$ as follows:

$$
\begin{aligned}
\gamma^{\prime} & = \begin{cases}\delta(t-v+l) & \text { for } v-l \leq t \leq v, \\
\bar{\gamma}(t) & \text { for } v \leq t \leq b-a+u,\end{cases} \\
\gamma^{\prime \prime} & = \begin{cases}\gamma(t) & \text { for } u \leq t \leq v, \\
\delta(v+l-t) & \text { for } v \leq t \leq v+l .\end{cases}
\end{aligned}
$$

In $\pi_{1}(X)$ we can write $\left[\gamma^{\prime}\right]=l^{\prime}\left[\sigma_{1}\right]+m^{\prime}\left[\sigma_{2}\right]$ and $\left[\gamma^{\prime \prime}\right]=l^{\prime \prime}\left[\sigma_{1}\right]+m^{\prime \prime}\left[\sigma_{2}\right]$. From the relations

$$
\left[\gamma^{\prime}\right] \cap\left[\gamma^{\prime}\right]=\left[\gamma^{\prime \prime}\right] \cap\left[\gamma^{\prime \prime}\right]=\left[\gamma^{\prime}\right] \cap\left[\gamma^{\prime \prime}\right]=\left[\gamma^{\prime}\right] \cap[\gamma]=\left[\gamma^{\prime \prime}\right] \cap[\gamma]=0,
$$

and since $\gamma^{\prime}$ and $\gamma^{\prime \prime}$ are simple, it follows that $m^{\prime}=m^{\prime \prime}=0$ and $\left\{l^{\prime}, l^{\prime \prime}\right\} \subseteq\{0,1\}$, which shows that one of the curves $\gamma^{\prime}, \gamma^{\prime \prime}$ is a $\operatorname{vh}_{\sigma}$-curve and the other, say $\gamma^{\prime \prime}$, is a null-homotopic left-turning curve. The turning-points $\gamma^{\prime \prime}\left(t_{k}\right), q, p, \gamma^{\prime \prime}\left(t_{1}\right)$ belong to two consecutive left double-turns of $\gamma^{\prime \prime}$ that may or may not be unobstructed. By Lemma 3.31, $\gamma^{\prime \prime}$ has at least two other $\gamma^{\prime \prime}$-unobstructed left double-turns, and these remain unobstructed when considered as left double-turns of $\gamma$.

From Lemma 3.28 we get the following important result.

COROLlary 3.33. Any simple closed vh-curve of order at least four has at least one unobstructed tag in each direction.

Definition 3.34. A $\operatorname{vh}_{\sigma}$-domain $D=D\left(\gamma, \gamma^{\prime}\right)$ is simple if $\operatorname{ord}(\gamma)=$ $\operatorname{ord}\left(\gamma^{\prime}\right)$ and, possibly after re-indexing, $\operatorname{shape}(\gamma)=\operatorname{shape}\left(\gamma^{\prime}\right)$, each pair of turning-point $\gamma\left(t_{i}\right), \gamma^{\prime}\left(t_{i}\right)$ can be joined by a straight line, whose interior lies entirely in $D$.

Definition 3.35. A $\mathrm{vh}_{\sigma}$-domain will be called elementary if cutting off the shadow of a tag or stair-like turn leaves a simple $\mathrm{vh}_{\sigma}$-domain. There are three types of elementary $\mathrm{vh}_{\sigma}$-domains, shown in Figure 4.

Definition 3.36. A $\mathrm{vh}_{\sigma}$-domain $D=D\left(\gamma, \gamma^{\prime}\right)$ is left reduced if $\gamma^{\prime}$ has no $\left\{\gamma, \gamma^{\prime}\right\}$-unobstructed right tags and no $\left\{\gamma, \gamma^{\prime}\right\}$-unobstructed right stair-like turning-points. It is right reduced if $\gamma$ has no $\left\{\gamma, \gamma^{\prime}\right\}$-unobstructed left tags 
and no $\left\{\gamma, \gamma^{\prime}\right\}$-unobstructed left stair-like turns. If it is both right and left reduced, it is simply reduced.

Lemma 3.37. Any reduced $v h_{\sigma}$-domain is simple.

Proof. Note that if a double-turn is primary-obstructed by a turningpoint, this turning-point is part of a double-turn. Let $d^{\prime}$ be the middle leg of a double-turn primary-obstructing a left double-turn of $\gamma$, with middle leg $d$. Assume that $d^{\prime}$ is a leg of $\gamma$. Then since $D$ lies to the left of $\gamma, d$ and $d^{\prime}$ have opposite directions. A perpendicular line-segment $e$ joining $d$ and $d^{\prime}$ to the left of both will cut the domain $D$ into two disjoint domains with $e$ as the common boundary. One of these domains is contractible and is bounded by a left-turning curve. By Lemma 3.31 this boundary has at least four unobstructed left double-turns, and at most two of them involve the leg $e$, showing that $\gamma$ has an unobstructed left double-turn. By Lemma 3.28 it also has an unobstructed left tag. This is a contradiction since $D$ is reduced. It follows that $d^{\prime}$ is the middle leg of a left double-turn of $\gamma^{\prime}$. Similarly, every right double-turn of $\gamma^{\prime}$ is primary-obstructed by a right double-turn of $\gamma$. We have shown that we have partial surjections

$$
\operatorname{Double}_{l}\left(\gamma^{\prime}\right) \rightarrow \operatorname{Double}_{l}(\gamma) \text { and } \operatorname{Double}_{r}(\gamma) \rightarrow \operatorname{Double}_{r}\left(\gamma^{\prime}\right) .
$$

Since a $\mathrm{vh}_{\sigma}$-curve has the same number of right turning-points and left turning-points, it follows from Lemma 3.17 that the partial surjections above are bijections, and hence if a left stair-like turning-point of $\gamma$ is primaryobstructed by a turning-point $p^{\prime}$, then $p^{\prime}$ is a left stair-like turning-point of $\gamma^{\prime}$. Therefore we have partial surjections of stair-like turning-points as well,

$$
\operatorname{Stair}_{l}\left(\gamma^{\prime}\right) \rightarrow \operatorname{Stair}_{l}(\gamma) \text { and } \operatorname{Stair}_{r}(\gamma) \rightarrow \operatorname{Stair}_{r}\left(\gamma^{\prime}\right)
$$

By cutting $D$ at the end and at the beginning of consecutive strings of length at least two of similar turns of $\gamma$, reducedness shows that $\gamma$ and $\gamma^{\prime}$ have equal derived shapes, $\operatorname{ds}([\gamma])=\mathrm{ds}\left(\left[\gamma^{\prime}\right]\right)$. By Lemma 3.16,

$$
\left|\operatorname{Stair}_{r}(\gamma)\right|-\left|\operatorname{Stair}_{l}(\gamma)\right|=\left|\operatorname{Stair}_{r}\left(\gamma^{\prime}\right)\right|-\left|\operatorname{Stair}_{l}\left(\gamma^{\prime}\right)\right| .
$$

It follows that primary obstruction is a bijection of stair-like turns as well. Therefore primary obstruction is a bijection between all turns of $\gamma$ and $\gamma^{\prime}$, and hence $D$ is simple.

Definition 3.38. A vh-roof or vh-fibration for a $v_{\sigma}$-domain $D=$ $D\left(\gamma, \gamma^{\prime}\right)$ is a continuous function $f: \bar{D} \rightarrow[0,1]$ with the following properties:

(1) For all $t, f(\gamma(t))=0$ and $f\left(\gamma^{\prime}(t)\right)=1$.

(2) For all $s \in[0,1]$, the fiber $f^{-1}(s)$ is the image of a simple $v_{\sigma^{-}}$-curve.

(3) There is a subdivision of $D$ into trapeziums and triangles on which the restriction of $f$ is linear.

(4) The order-function ord $\left(f^{-1}(-)\right):[0,1] \rightarrow \mathbb{N}$ is lower semicontinuous. 
(5) There is a sequence $0=r_{0}<r_{1}<\cdots<r_{n}=1$ such that $\operatorname{ord}\left(f^{-1}(-)\right)$ is constant on the interval $\left\langle r_{i}, r_{i+1}\right\rangle$ for $0 \leq i<n$.

A vh-roof will be called order-reducing if in addition it satisfies:

(6) If $m=\min \left\{\operatorname{ord}\left(f^{-1}(s)\right) \mid s \in[0,1]\right\}$, the set $\left\{s \mid \operatorname{ord}\left(f^{-1}(s)\right)=m\right\}$ is an interval $[a, b] \subseteq[0,1]$, which is either equal to $[0,1]$ or contained in $\langle 0,1\rangle$. In the latter case the order-function $\operatorname{ord}\left(f^{-1}(-)\right)$ is monotone on both $[0, a]$ and $[b, 1]$.

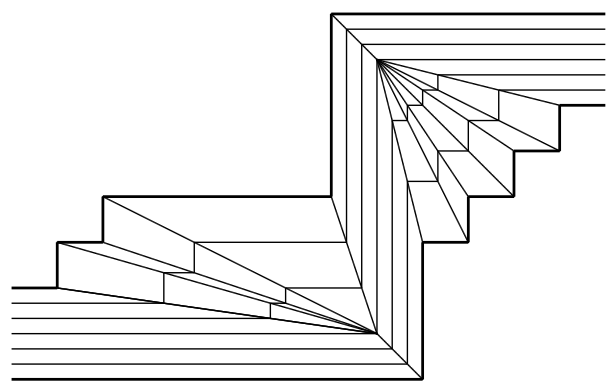

Fig. 3. Part of an order-reducing vh-roof

Note that by lower semicontinuity and monotonicity, the order-function of an order-reducing vh-roof is constant in a neighborhood of $\{0,1\}$.

THEOREM 3.39. Every vh-domain has an order-reducing vh-roof.

Proof. If $D$ is simple, the function which is linear on each trapezium of the domain is an order-reducing roof. If $D$ is not simple, we may assume that the right boundary $\gamma$ has an unobstructed left tag or a stair-like turn. We cut off the shadow of this tag or stair-like turn and obtain a domain $D^{\prime}$ of lower order. By induction $D^{\prime}$ has an order-reducing vh-roof $f^{\prime}$. Let $\gamma_{s}$ be the $\mathrm{vh}_{\sigma}$-curve with image the fiber $f^{\prime-1}(s)$. If $k>0$ is so small that the domain $E^{\prime}=D\left(\gamma_{0}, \gamma_{k}\right)$ is simple, and $g$ is a vh-roof on the elementary domain $E=D \backslash D\left(\gamma_{k}, \gamma_{1}\right)=D\left(\gamma, \gamma_{k}\right)$, as shown in Figure 4, the function $f$ defined by $f(x)=\left(f^{\prime}(x)+k\right) /(1+k)$ for $x \in D\left(\gamma_{k}, \gamma\right)$ and $k \cdot g(x) /(1+k)$ for $x \in E$ is an order-reducing vh-roof on $D$.
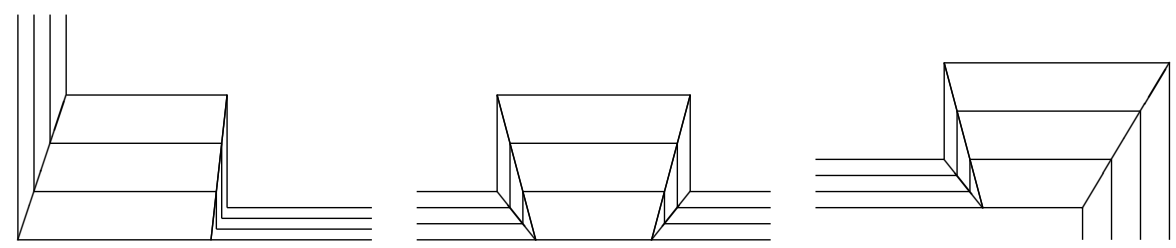

Fig. 4. Vh-roofs on elementary domains in the case of a stair-like turn, symmetric tag and asymmetric tag 
4. Simple topological measures and vh-fibers. In this section we will show that the $\sigma$-component (Definition 4.2) of a simple topological measure $\mu$ is determined by its restriction to $v h_{\sigma}$-fibers, $\mathcal{V} h_{\sigma}$.

Since a curve is a function, and we are often interested only in the image of the curve, we make the following definition.

DeFinition 4.1. By a vh-fiber we shall simply mean the image of a vh-curve. We denote the set of all $v_{\sigma}$-fibers of $X$ by $\mathcal{V} h_{\sigma}$.

Note that if $K$ is a $\mathrm{vh}_{\sigma}$-fiber and $p \in K$ is a corner of $K$, there is a unique vh $_{\sigma}$-curve $\gamma$ with $\gamma(a)=p, \gamma \sim \sigma_{1}$ and $\gamma([a, b])=K$. If $K$ does not have corners we can start the parameterization anywhere. The important thing is the orientation, which enables us to define left and right corners and, up to circular equivalence, the shape of a $\mathrm{vh}_{\sigma}$-fiber.

Recall that $\mathcal{A}_{\sigma}$ denotes the subfamily of the open or closed sets that are $\sigma$-sets (see Definition 1.7).

Definition 4.2. For any topological measure $\mu$ on the torus $X$, we denote by $\mu_{\sigma}$ the restriction of $\mu$ to the sets $\mathcal{A}_{\sigma}$, and call it the $\sigma$-component of $\mu$.

Proposition 4.3. The $\sigma$-component of a simple topological measure is determined by its restriction to $\mathcal{V} h_{\sigma}$.

Proof. Let $\theta$ and $\theta^{\prime}$ be simple topological measures whose restrictions to $\mathcal{V} h_{\sigma}$ coincide. By additivity and symmetry it suffices to show that if $\theta(U)=$ 1 , then $\theta^{\prime}(U)=1$ for any $U \in \mathcal{U}_{\sigma}$, the open $\sigma$-sets. By Definition 0.1(b), choose a solid closed $\sigma$-set $F \subseteq U$ with $\theta(F)=1$. By compactness we can cover $F$ with a finite number of open rectangles with sides parallel to the coordinate axes and with closures lying in $U$. Their union is then a vh $_{\sigma}$-domain $D=D\left(\gamma, \gamma^{\prime}\right)$, with $\bar{D} \subseteq U$. If $f: \bar{D} \rightarrow[0,1]$ is a reducing roof, there is a unique fiber $f^{-1}(t)$ with $\theta\left(f^{-1}(t)\right)=1$. The measures $\theta$ and $\theta^{\prime}$ agree on $f^{-1}(t)$, and by monotonicity $\theta^{\prime}(U)=1$.

COROLlary 4.4. In order to show Theorem 1.9, it suffices to show that the family $\mathcal{V} h_{\sigma, \mu}$ of $v h_{\sigma}$-fibers with $\mu$-measure equal to one is liftable.

5. Special domains. In this section we study $\mathrm{vh}_{\sigma^{-}}$-curves and $\operatorname{vh}_{\sigma^{-}}$ domains on the cylinder $Z$ covering the torus $X$. The main result is a criterion for specialty, which will be used in Lemma 9.8, the final lemma in the proof of the lifting conjecture. Except for the definition of the various metrics, the reader may skip this section until then.

We let $q: Z \rightarrow X$ be the covering map. The coordinates on $Z$ are $\left(\widetilde{x}_{1}, x_{2}\right)$ and when $\sigma_{2}$ acts on $Z$ as a covering transformation, we have $x_{2}\left(\left[\sigma_{2}\right](z)\right)=$ $x_{2}(z)+1$. 
Definition 5.1. A simply closed curve $\widetilde{\gamma}:[a, b] \rightarrow Z$ will be called a $v h$ curve if the composition $\gamma=q \circ \widetilde{\gamma}$ satisfies all the conditions of Definition 3.3, except it need not be simply closed. We say that a vh-curve $\widetilde{\gamma}$ is a $v h_{\sigma}$-curve if its projection is homotopic to $\sigma_{1}$. A vh-curve $\widetilde{\gamma}$ will be called special if $q \circ \widetilde{\gamma}$ is simply closed. We make the same definition for fibers on $Z$. We denote by $\widetilde{\mathcal{V h}}_{\sigma}$ the set of all special $v \mathrm{vh}_{\sigma}$-fibers in $Z$. The order-function defines gradings on both $\mathcal{V} h_{\sigma}$ and $\widetilde{\mathcal{V}} h_{\sigma}$. We write $\mathcal{V} h_{\sigma}^{(2 n)}$ for the set of $v h_{\sigma}$-fibers of order not exceeding $2 n$. Each $\operatorname{vh}_{\sigma}$-fiber $\widetilde{K}$ on $Z$ divides $Z$ into two disjoint sets: the points to the right of $\widetilde{K}$, denoted by $R(\widetilde{K})=\{z \mid z<\widetilde{K}\}$, and the points to the left of $\widetilde{K}$, denoted by $L(\widetilde{K})=\{z \mid z>\widetilde{K}\}$.

Note that the group $\pi_{1}(X) / \sigma \approx \mathbb{Z}$ acts on $\widetilde{\mathcal{V}}{ }_{\sigma}$, and $\mathcal{V} h_{\sigma}$ is the quotient of the set of special fibers under this action. For any $\operatorname{vh}_{\sigma}$-fiber $\widetilde{K}, Z=$ $R(\widetilde{K}) \cup \widetilde{K} \cup L(\widetilde{K})$.

For each vh-curve $\gamma:[a, b] \rightarrow X$ and each point $z \in q^{-1}(\gamma(a))$ we have a unique commutative diagram

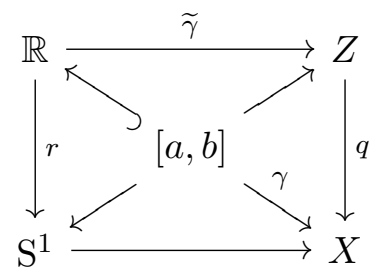

where $r$ is the covering map $r(t)=\exp \left(2 \pi i \frac{t-a}{b-a}\right)$ and $\widetilde{\gamma}(a)=z$. We call $\widetilde{\gamma}$ the lift of $\gamma$ determined by the choice of the starting point $z \in q^{-1}(\gamma(a))$.

Lemma 5.2. If $\widetilde{\gamma}:[a, b] \rightarrow Z$ is a special vh-curve, then $\gamma=q \circ \widetilde{\gamma}$ is either homotopically trivial or a vh ${ }_{\sigma}$-curve.

Proof. If the homotopy class $[\gamma]$ is not in $\sigma$ and $\widetilde{\gamma}: \mathbb{R} \rightarrow Z$ is the lift as defined by the commutative diagram (5.1), we have $\widetilde{\gamma}(a) \neq \widetilde{\gamma}(b)$, and this is a contradiction because $\widetilde{\gamma}$ is a closed curve.

Definition 5.3. Let $\widetilde{K}<\widetilde{K}^{\prime}$ be special $v_{\sigma}$-fibers on $Z$. We call the domain between them a $v h_{\sigma}$-domain on $Z$, and denote it by $\widetilde{D}\left(\widetilde{K}, \widetilde{K}^{\prime}\right)$. Even though the boundaries of a $v_{\sigma}$-domain $\widetilde{D}$ are special, the restriction to $\widetilde{D}$ of the projection $q$ may not be one-to-one. We say that a $v_{\sigma}$-domain $\widetilde{D}$ is special if $\left.q\right|_{\widetilde{D}}: \widetilde{D} \rightarrow q(\widetilde{D})$ is a homeomorphism.

Definition 5.4. For any subset $\widetilde{D} \subseteq Z$, we denote by $\widetilde{D}[1]$ the set $\left[\sigma_{2}(\widetilde{D})\right]$. Repeated actions of $\left[\sigma_{2}\right]$ and its inverse yield the sets $\widetilde{D}[n]$ for every integer $n$.

For any special $\operatorname{vh}_{\sigma}$-fiber $\widetilde{K}$, the domain $\widetilde{D}(\widetilde{K}, \widetilde{K}[1])$ is a fundamental domain for the action of $\left[\sigma_{2}\right]$, and we have the following result. 
Lemma 5.5. The domain $\widetilde{D}=\widetilde{D}\left(\widetilde{K}, \widetilde{K}^{\prime}\right)$ is special if and only if both $\widetilde{K}$ and $\widetilde{K}^{\prime}$ are special, and $\widetilde{K}^{\prime} \leq \widetilde{K}[1]$.

In order to state our main specialty criterion and also for various purposes later on, we need to use convenient metrics on the spaces $Y, Z, X, \mathcal{V} h_{\sigma}$ and $\widetilde{\mathcal{V}}_{\sigma}$, depending on the choice of $\left(\sigma_{1}, \sigma_{2}\right)$-coordinates. We will simply write $d$ for these metrics since there can be no ambiguity.

Let $\widetilde{\sigma}_{1}:[0,1] \rightarrow Z$ be the lift of $\sigma_{1}$ given by $\mathbf{x}_{2}\left(\widetilde{\sigma}_{1}(t)\right)=0$. For a set $\widetilde{K} \in \widetilde{\mathcal{V h}}_{\sigma}$ and a point $\widetilde{q} \in \widetilde{K}$, let $\widetilde{\gamma}(\widetilde{K}, \widetilde{q}):[0,1] \rightarrow Z$ be the parameterization of $\widetilde{K}$ starting at $\widetilde{q}$, with uniform speed and the direction making $\widetilde{\gamma}(\widetilde{K}, \widetilde{q}) \sim \widetilde{\sigma}_{1}$.

Definition 5.6. For pairs of points $\left(r_{1}, r_{2}\right) \in Y \times Y,\left(q_{1}, q_{2}\right) \in Z \times Z$ and $\left(p_{1}, p_{2}\right) \in X \times X$, we define

$$
\begin{aligned}
& d\left(r_{1}, r_{2}\right)=\max \left\{\left|\mathbf{x}_{1}\left(r_{1}\right)-\mathbf{x}_{1}\left(r_{2}\right)\right|,\left|\mathbf{x}_{2}\left(r_{1}\right)-\mathbf{x}_{2}\left(r_{2}\right)\right|\right\}, \\
& d\left(q_{1}, q_{2}\right)=\min \left\{d\left(r_{1}, r_{2}\right) \mid r_{1} \in r^{-1}\left(q_{1}\right) \text { and } r_{2} \in r^{-1}\left(q_{2}\right)\right\}, \\
& d\left(p_{1}, p_{2}\right)=\min \left\{d\left(r_{1}, r_{2}\right) \mid r_{1} \in p^{-1}\left(p_{1}\right) \text { and } r_{2} \in p^{-1}\left(p_{2}\right)\right\} .
\end{aligned}
$$

For continuous functions $\widetilde{\gamma}_{1}, \widetilde{\gamma}_{2}:[0,1] \rightarrow Z$ we define

$$
d\left(\widetilde{\gamma}_{1}, \widetilde{\gamma}_{2}\right)=\max \left\{d\left(\widetilde{\gamma}_{1}(t), \widetilde{\gamma}_{2}(t)\right) \mid t \in[0,1]\right\} .
$$

For pairs of $\operatorname{vh}_{\sigma}$-fibers $\left(\widetilde{K}_{1}, \widetilde{K}_{2}\right) \in \widetilde{\mathcal{V}}_{\sigma} \times \widetilde{\mathcal{V}}_{\sigma}$ and $\left(K_{1}, K_{2}\right) \in \mathcal{V} h_{\sigma} \times \mathcal{V} h_{\sigma}$, we will use the Fréchet-like distances defined by

$$
\begin{aligned}
& d_{F}\left(\widetilde{K}_{1}, \widetilde{K}_{2}\right)=\min \left\{d\left(\widetilde{\gamma}_{(}\left(\widetilde{K}_{1}, \widetilde{q}_{1}\right), \widetilde{\gamma}\left(\widetilde{K}_{2}, \widetilde{q}_{2}\right)\right) \mid \widetilde{q}_{1} \in \widetilde{K}_{1}, \widetilde{q}_{2} \in \widetilde{K}_{2}\right\}, \\
& d_{F}\left(K_{1}, K_{2}\right)=\min \left\{d\left(\widetilde{K}_{1}, \widetilde{K}_{2}\right) \mid\left(\widetilde{K}_{1}, \widetilde{K}_{2}\right) \text { a lift of }\left(K_{1}, K_{2}\right)\right\} .
\end{aligned}
$$

For a point $p$ and a compact set $K$, we define

$$
d(p, K)=\min \left\{d\left(p, p^{\prime}\right) \mid p^{\prime} \in K\right\} .
$$

This distance is used to fatten up sets. For any $\varepsilon>0$, and any set $D$, we call the set $D_{\varepsilon}=\{p \mid d(p, D)<\varepsilon\}$ the $\varepsilon$-fattening of $D$. For compact sets $K_{1}$ and $K_{2}$, the Hausdorff distance is

$$
d_{\mathrm{H}}\left(K_{1}, K_{2}\right)=\max \left\{d_{\mathrm{asym}}\left(K_{1}, K_{2}\right), d_{\text {asym }}\left(K_{2}, K_{1}\right)\right\},
$$

where

$$
d_{\text {asym }}\left(K_{1}, K_{2}\right)=\sup \left\{d\left(p, K_{2}\right) \mid p \in K_{1}\right\}=\inf \left\{\varepsilon \mid K_{1} \subseteq\left(K_{2}\right)_{\varepsilon}\right\} .
$$

Lemma 5.7. All of the above functions are metrics.

Proof. Note that if $\left(p_{1}, p_{2}\right) \in X \times X$, and $r_{2} \in Y$ is any point in the fiber $p^{-1}\left(p_{2}\right)$, then $d\left(p_{1}, p_{2}\right)=\min \left\{d\left(r_{1}, r_{2}\right) \mid r_{1} \in p^{-1}\left(p_{1}\right)\right\}$. 
REMARK 5.8. Note that when we restrict ourselves to fibers of order $<n$, the Hausdorff and Fréchet metrics are equivalent.

In the rest of this section we will study the fine structure of $\mathrm{vh}_{\sigma}$-domains in $Z$. Recall that they are of the form $\widetilde{D}=\widetilde{D}\left(\widetilde{K}, \widetilde{K}^{\prime}\right)$ with $\widetilde{K}<\widetilde{K}^{\prime}$ and with both boundaries special. Our main result is that an $(\varepsilon, \delta)$-immobile, reduced $\mathrm{vh}_{\sigma}$-domain is special.

Definition 5.9. Let $\widetilde{K}$ be a special $\operatorname{vh}_{\sigma}$-fiber and let $d_{\widetilde{K}}: Z \rightarrow \mathbb{R}$ denote the distance from $\widetilde{K}$. A number $\varepsilon>0$ will be called left admissible for $\widetilde{K}$ if for every $\delta<\varepsilon$, the set $\widetilde{K}_{\delta^{+}}=L(\widetilde{K}) \cap d_{\widetilde{K}}^{-1}(\delta)$ is a special $\operatorname{vh}_{\sigma^{-}}$fiber, and the domain $\widetilde{D}\left(\widetilde{K}, \widetilde{K}_{\delta^{+}}\right)$is special. The least upper bound of the $\widetilde{K}$ left admissible numbers is called the left fattening number of $\widetilde{K}$.

We define the the right fattening number of $\widetilde{K}$ similarly.

An admissible number for $\widetilde{K}$ is a number $\varepsilon$ which is both left and right admissible, and such that for each $\delta<\varepsilon$, the $\delta$-fattening $\widetilde{K}_{\delta}=\widetilde{D}\left(\widetilde{K}_{\delta^{-}}, \widetilde{K}_{\delta^{+}}\right)$ is special. The least upper bound of the $\widetilde{K}$ admissible numbers is called the fattening number of $\widetilde{K}$. We denote these numbers by $\varepsilon_{l}(\widetilde{K}), \varepsilon_{r}(\widetilde{K})$ and $\varepsilon(\widetilde{K})$.

The reader may show that $\varepsilon(\widetilde{K})=\frac{1}{2} \min \left\{\varepsilon_{r}(\widetilde{K}), \varepsilon_{l}(\widetilde{K})\right\}$.

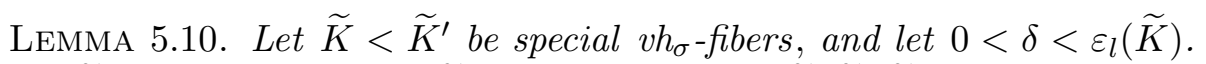
If $d(z, \widetilde{K}) \leq \delta$ for every $z \in \widetilde{K}^{\prime}$, then the domain $\widetilde{D}\left(\widetilde{K}, \widetilde{K}^{\prime}\right)$ is special.

Note that we have a similar result using the right fattening number of $\widetilde{K}^{\prime}$.

Since any special fiber is a lifting of a $v_{\sigma}$-fiber on $X$, the fattening numbers are defined for fibers $K \subseteq X$. In order to find the fattening numbers without using lifts, we can do the following. It will be convenient to think in a non-standard way. On the non-standard torus, we let a fiber $K$ consist of two disjoint fibers $K^{l}$ and $K^{r}$ of a non-standard distance less than any standard positive number. The points of $K^{l}$ will be called the left points of $K$, and $K^{r}$ consists of the right points of $K$. Two points $p$ and $p^{\prime}$ of $K$ will be called joinable if they belong to different parallel legs, possibly of opposite direction, and they can be joined by a line-segment meeting $K$ only in $p$ and $p^{\prime}$. Using the joinability concept we can define the following numbers:

$$
\begin{aligned}
& \varepsilon_{l l}(K)=\inf \left\{d\left(p, p^{\prime}\right) \mid p \in K^{l}, p^{\prime} \in K^{l}, \text { and }\left(p, p^{\prime}\right) \text { joinable }\right\}, \\
& \varepsilon_{l r}(K)=\inf \left\{d\left(p, p^{\prime}\right) \mid p \in K^{l}, p^{\prime} \in K^{r}, \text { and }\left(p, p^{\prime}\right) \text { joinable }\right\}, \\
& \varepsilon_{r l}(K)=\inf \left\{d\left(p, p^{\prime}\right) \mid p \in K^{r}, p^{\prime} \in K^{l}, \text { and }\left(p, p^{\prime}\right) \text { joinable }\right\}, \\
& \varepsilon_{r r}(K)=\inf \left\{d\left(p, p^{\prime}\right) \mid p \in K^{r}, p^{\prime} \in K^{r}, \text { and }\left(p, p^{\prime}\right) \text { joinable }\right\} .
\end{aligned}
$$

Of course $\varepsilon_{l r}(K)=\varepsilon_{r l}(K)$. 
LEMma 5.11. We have the following local formulas for the fattening numbers:

$$
\varepsilon_{l}(K)=\min \left\{\varepsilon_{l r}(K), \frac{1}{2} \varepsilon_{l l}(K)\right\}, \quad \varepsilon_{r}(K)=\min \left\{\varepsilon_{r l}(K), \frac{1}{2} \varepsilon_{r r}(K)\right\} .
$$

The fattening numbers are positive since distinct parallel legs have positive distance. In order to prove Lemma 9.8, we need to generalize the primary obstruction relation on double-turns and stair-like turns.

Definition 5.12. Let $K$ be $\mathrm{a} \mathrm{vh}_{\sigma}$-fiber, and let $0 \leq \varepsilon<\varepsilon(K)$, the fattening number of $K$. Consider the two disjoint fibers $K_{\varepsilon^{+}}$and $K_{\varepsilon^{-}}$. We will say that a left (resp. right) double-turn or stair-like turn of $K$ is primary$\varepsilon$-obstructed if the corresponding turn of $K_{\varepsilon^{+}}$(resp. $K_{\varepsilon^{-}}$) is $\left\{K_{\varepsilon^{+}}, K_{\varepsilon^{-}}\right\}$obstructed.

Note that if $K$ is a $v_{\sigma}$-fiber and $0 \leq \varepsilon<\varepsilon_{l}(K)$, the primary $\varepsilon$ obstruction relation makes the double-turns of $K$ into a forest. The proof of Lemma 3.32 essentially gives us the following strengthening.

Lemma 5.13. Any vh-fiber $K$ of order at least four has at least one $\varepsilon$-unobstructed tag in each direction, provided that $\varepsilon<\varepsilon(K)$.

In particular, we observe the following.

Corollary 5.14. Let $K$ be a vh $h_{\sigma}$-fiber, and let $0<\varepsilon<\varepsilon(K)$. If $e$ is an $\varepsilon$-unobstructed tag of $K$, and if $K^{\prime}$ is the fiber obtained by chopping off the tag defined by $e$, then $\varepsilon \leq \varepsilon\left(K^{\prime}\right)$.

Definition 5.15. Let $\varepsilon>\delta>0$. A $\operatorname{vh}_{\sigma}$-domain $\widetilde{D}=\widetilde{D}\left(\widetilde{K}, \widetilde{K}^{\prime}\right)$ will be said to have the $(\varepsilon, \delta)$-property if the following holds:

1) $\varepsilon \leq \min \left\{\varepsilon(K), \varepsilon\left(K^{\prime}\right)\right\}$.

2) $\widetilde{K}_{\delta^{+}} \leq \widetilde{K}^{\prime}$.

3) Any double or stair-like turn of $K$ that is $\{K\}$-unobstructed is $\varepsilon$-unobstructed, and the same for double and stair-like turns of $K^{\prime}$.

Note that for any $\operatorname{vh}_{\sigma}$-domain $\widetilde{D}$, there are $\varepsilon>\delta>0$ such that $\widetilde{D}$ has the $(\varepsilon, \delta)$-property.

Definition 5.16. Let $\varepsilon>\delta>0$ and let $\widetilde{D}=\widetilde{D}\left(\widetilde{K}, \widetilde{K}^{\prime}\right)$ be a $\operatorname{vh}_{\sigma}$-domain with the $(\varepsilon, \delta)$-property. A leg of $K$ (resp. $\left.K^{\prime}\right)$ will be said to be movable if it can be moved a positive distance to the left (resp. right) without destroying the $(\varepsilon, \delta)$-property. The domain will be called $(\varepsilon, \delta)$-immobile if there are no movable legs.

Definition 5.17. A $\operatorname{vh}_{\sigma}$-domain $\widetilde{D}=\widetilde{D}\left(\widetilde{K}, \widetilde{K}^{\prime}\right)$ will be called left reduced if the lift of every $\left\{K^{\prime}\right\}$-unobstructed right tag and right stair-like turn of $K^{\prime}$ is $\{\widetilde{K}\}$-obstructed. It will be called right reduced if the lift of 
every $\{K\}$-unobstructed left tag and left stair-like right turn of $K$ is $\left\{\widetilde{K}^{\prime}\right\}$ obstructed. A domain on $Z$ will be called reduced if it is both left and right reduced.

Definition 5.18. A double-turn $e$ is snugly obstructed by a double-turn $d$ if $d$ is the only double-turn that primary-obstructs $e$ and meets the shadow of $e$.

A stair-like turn $e$ is snugly obstructed by a turning-point $d$ if $d$ is the only turning-point that primary-obstructs $e$ and meets the shadow of $e$.

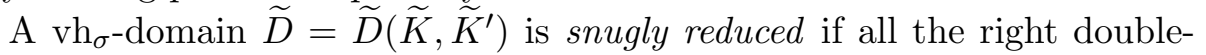
turns and stair-like turns of $K^{\prime}$ that are primary-obstructed by $K$ are snugly obstructed by $\widetilde{K}$, and the other way around.

Definition 5.19. A situation will be a 5 -tuple $s=\left(p, p^{\prime}, \widetilde{K}, \widetilde{K}^{\prime}, c\right)$, where $p$ and $p^{\prime}$ are points in $X$ that have lifts $\widetilde{p}$ and $\widetilde{p}^{\prime}$ to the special $\operatorname{vh}_{\sigma}$-fibers $\widetilde{K}$ and $\widetilde{K}^{\prime}$. The points $p$ and $p^{\prime}$ belong to the interiors of parallel legs $e$ and $e^{\prime}$ of the projected fibers $K$ and $K^{\prime}$, and $c$ is a directed line-segment containing the points $p$ and $p^{\prime}$, meeting the legs they belong to transversally, and meeting $p$ before $p^{\prime}$.

Definition 5.20. The direction of a situation $s=\left(p, p^{\prime}, \widetilde{K}, \widetilde{K}^{\prime}, c\right)$ is the pair $\operatorname{dir}(s)=\left(\operatorname{dir}(c, e), \operatorname{dir}\left(c, e^{\prime}\right)\right) \in\{r, l\}^{2}$, where $e$ and $e^{\prime}$ are the legs that $p$ and $p^{\prime}$ belong to.

Definition 5.21. The level of a situation $s=\left(p, p^{\prime}, \widetilde{K}, \widetilde{K}^{\prime}, c\right)$ is the integer $k$ with the property that if $\widetilde{p}, \widetilde{p}^{\prime}$ and $\widetilde{c}$ are lifts to $Z$ of $p, p^{\prime}$ and $c$ with $\widetilde{p} \in \widetilde{K}, \widetilde{p}^{\prime} \in \widetilde{K}^{\prime}$ and $q(\widetilde{c})=c$, then $\widetilde{p}^{\prime} \in \widetilde{c}[k]$.

We have the following very basic lemmas.

Lemma 5.22. For situations $s=\left(p, p^{\prime}, \widetilde{K}, \widetilde{K}^{\prime}, c\right), s^{\prime}=\left(p^{\prime}, p^{\prime \prime}, \widetilde{K}^{\prime}, \widetilde{K}^{\prime \prime}, c\right)$ and $s^{\prime \prime}=\left(p, p^{\prime \prime}, \widetilde{K}, \widetilde{K}^{\prime \prime}, c\right)$, where $p, p^{\prime}$ and $p^{\prime \prime}$ are three points on the segment $c$, we have

$$
\operatorname{level}\left(s^{\prime \prime}\right)=\operatorname{level}(s)+\operatorname{level}\left(s^{\prime}\right) .
$$

LEMMA 5.23. If in the situation $s=\left(p, p^{\prime}, \widetilde{K}, \widetilde{K}, c\right), p$ and $p^{\prime}$ are nearest neighbors on $c$, then the level is determined by the direction only, and we have

$$
\operatorname{level}(s)= \begin{cases}1 & \text { if } \operatorname{dir}(s)=l l, \\ 0 & \text { if } \operatorname{dir}(s) \in\{l r, r l\}, \\ -1 & \text { if } \operatorname{dir}(s)=r r .\end{cases}
$$

Proof. The case $r l$ has been treated in the proof of Lemma 3.32. The case $l r$ is similar. In the case $l l$, let $\alpha=\gamma\left(K, p^{\prime}, p\right) * \gamma\left(c, p, p^{\prime}\right)$ and $\beta=$ $-\gamma\left(c, p^{\prime}, p\right) * \gamma\left(K, p, p^{\prime}\right)$. Then as $K$ has no self-intersections we get $[\alpha] *[\beta]$ $=[\gamma(K)]=\left[\sigma_{1}\right]$, and the intersection number $[\alpha] \cap[\beta]$ is 1 . It follows that 
$\alpha \sim(1-m) \sigma_{1}-\sigma_{2}$ and $\beta \sim m \sigma_{1}+\sigma_{2}$, showing that $\widetilde{p}^{\prime} \in \widetilde{c}[1]$. We leave the case $r r$ to the reader.

Lemma 5.24. Let $\widetilde{K}<\widetilde{K}^{\prime}$ be vh-fibers and let $c$ be a directed linesegment on $X$ meeting both $K$ and $K^{\prime}$ transversally. Let $p_{1}, p_{2} \in K$ and $p_{2}^{\prime} \in K^{\prime}$ be points in $c$ with $p_{1}<p_{2}<p_{2}^{\prime}$. Assume that both situations $s_{1}=$ $\left(p_{1}, p_{2}, \widetilde{K}, \widetilde{K}, c\right)$ and $s_{2}=\left(p_{2}, p_{2}^{\prime}, \widetilde{K}, \widetilde{K}^{\prime}, c\right)$ have direction $r r$, that $p_{1}$ and $p_{2}$ are nearest neighbors, and that level $\left(s_{2}\right)=0$. Then there is at least one point $p_{1}^{\prime}$ with $p_{1}<p_{1}^{\prime}<p_{2}^{\prime}$ and with level $\left(s_{3}\right)=0$, where $s_{3}=\left(p_{1}, p_{1}^{\prime}, \widetilde{K}, \widetilde{K}^{\prime}, c\right)$.

Proof. We may assume that $c$ starts at $p_{1}$. If necessary we extend $c$, without making a loop, so that its lift $\widetilde{c}$ and its translations $\widetilde{c}[k]$ contain all intersection points with $\widetilde{K}$ and $\widetilde{K}^{\prime}$. Moving along $\widetilde{c}$ from $\widetilde{p}_{1}$, where we enter $\widetilde{D}=\widetilde{D}\left(\widetilde{K}, \widetilde{K}^{\prime}\right)$, we have to eventually get out of the domain again, so let $\widetilde{p}_{1}^{\prime}$ be the first point where we leave the domain again, and let $p_{1}^{\prime}$ be the projection. (Note that if $c$ makes a rational angle with the coordinate axis we may have to extend $\widetilde{c}$ to get out of the domain.) By additivity (Lemmas 5.22 and 5.23) it follows that if on $c$ we have $p_{1}<p_{2}<p_{2}^{\prime}<p_{1}^{\prime}$ and $p_{2}^{\prime}$ and $p_{1}^{\prime}$ are nearest $K^{\prime}$-neighbors, then $(-1)+0+(-1)=0$. This is impossible, so either $p_{1}<p_{1}^{\prime}<p_{2}^{\prime}$ or there are at least two points of $K^{\prime}$ of the opposite direction between $p_{2}^{\prime}$ and $p_{1}^{\prime}$. Let $q^{\prime}$ be such a point with $\operatorname{dir}\left(c, q^{\prime}\right)=l$ and $\operatorname{level}\left(p_{1}, q^{\prime}, \widetilde{K}, \widetilde{K}^{\prime}, c\right)=0$. But then the line-segment $\widetilde{d}$ enters $\widetilde{D}$ at $\widetilde{q}^{\prime}$ before it has left it, which is impossible, and this proves the lemma.

Lemma 5.25. An $(\varepsilon, \delta)$-immobile, snugly reduced vh ${ }_{\sigma}$-domain is special.

Proof. By assumption $\widetilde{K}^{\prime}$ forms the left border of every double or stairlike left turn of $\widetilde{K}_{\delta^{+}}$corresponding to $\{K\}$-unobstructed left double or stairlike turns of $K$, and vice versa. We have to check this property for obstructed ones as well. Let $p$ be a left turning-point of $K$, obstructed by a right turningpoint $q$ of $K$, and let $c$ be a directed line-segment joining $p$ and $q$. At least one of the curves $\alpha=\gamma(K, p, q) *-\gamma(c, q, p)$ and $\beta=\gamma(c, p, q) * \gamma(K, q, p)$ is a left loop, so by Lemmas 3.31 and 3.28 , slightly modified, we see that there is a $\{K\}$-unobstructed left tag of $K$ inside the loop. By our assumption this tag is snugly obstructed by $K^{\prime}$ and cannot get in there without crossing $c$ at least twice, in such a way that all situations that arise have level 0. Since $\widetilde{K}$ and $\widetilde{K}^{\prime}$ enclose a domain, the part of $K^{\prime}$ that is inside the loop $\alpha$ or $\beta$ is a left-turning loop, which shows that $p$ is obstructed by a left turning-point $p^{\prime}$ of $K^{\prime}$. By our assumptions the obstruction is snug and $d\left(p, p^{\prime}\right)=\delta$. Consider the case when $p$ is primary-obstructed by another left turning-point $q$ of $K$; we will prove that $p$ is snugly obstructed by a left turning-point of $K^{\prime}$ at distance $\delta$. Since a chain of such left-turning obstructions must terminate, we only have to prove that if $q$ is snugly obstructed by a left turning-point of $K^{\prime}$ at distance $\delta$, so is $p$. Let $q^{\prime}$ be the left turning-point of $K^{\prime}$ obstructing 
$q$ at distance $\delta$. By Lemma 5.24, there is a point $p^{\prime}$ of $K^{\prime}$ between $p$ and $q^{\prime}$. But since $\varepsilon>\delta$, it is in fact on the right side of $q$, which shows that $K^{\prime}$ primary-obstructs $p$, and by the assumptions this obstruction is snug and at distance $\delta$. A completely symmetric argument shows that all right turning-points of $K^{\prime}$ are snugly obstructed by right turning-points of $K$ at distance $\delta$. Since the number of left turning-points equals the number of right turning-points for both fibers, it follows that $\widetilde{K}^{\prime}=\widetilde{K}_{\delta^{+}}$. and the proposition follows from Lemma 5.10.

6. Pseudo-vh ${ }_{\sigma}$-domains and pseudo-roofs. The ideas of this chapter are quite simple and elementary, but a bit complicated to write down. To make it as simple as possible we were forced to invent a number of terms. We advice the reader to make her/his own drawings to see what is going on. The main theme in this section is about building pseudo-roofs over pseudo$\mathrm{vh}_{\sigma}$-domains. The main theorem is that order-reducing pseudo-roofs always exist, and can be approximated by regular order-reducing roofs of the same order. We also prove the crucial Lemma 6.38 about rightmost and leftmost fibers of directed pseudo-vh $\sigma^{\text {-domains. For }} \mathrm{vh}_{\sigma}$-fibers on $X$ we have a reflexive relation which we will denote by $\leq$ even though it is neither symmetric nor antisymmetric.

Definition 6.1. We will say that $K_{1} \leq K_{2}$ if there is an open solid vh $_{\sigma}$-domain $U \subseteq X$ such that $K_{1}$ forms the left boundary and $K_{2}$ forms the right boundary of $U$.

Definition 6.2. For fibers $K_{1}$ and $K_{2}$ with $K_{1} \leq K_{2}$, we denote by $D\left(K_{1}, K_{2}\right)$ the solid closed $\mathrm{vh}_{\sigma}$-set $X \backslash U$, where $U$ is the domain in Definition 6.1. We will call such a set a pseudo-vh ${ }_{\sigma}$-domain. If $D=D\left(K_{1}, K_{2}\right)$, we denote by $\mathcal{V} h_{\sigma}(D)$ the set of $\operatorname{vh}_{\sigma}$-fibers in $D$. Note that the restriction of $\leq$ to $\mathcal{V} h_{\sigma}(D)$ is a partial order.

Note that a pseudo-vh $\mathrm{vh}_{\sigma}$-domain is the closure of a $\mathrm{vh}_{\sigma}$-domain if and only if its complement is a proper $\mathrm{vh}_{\sigma}$-domain.

Definition 6.3. For any pseudo-vh $\sigma^{\text {-domain }} D=D\left(K_{1}, K_{2}\right)$, we define the order to be the sum of the orders of $K_{1}$ and $K_{2}$.

Definition 6.4. For any pseudo-vh ${ }_{\sigma}$-domain $D=D\left(K_{1}, K_{2}\right)$, the connected components of the interior of $D$ will be called the islands of $D$.

Definition 6.5. A pseudo-vh $\sigma^{\text {-domain }} D=D\left(K_{1}, K_{2}\right)$ will be called simple if there is an $\varepsilon>0$ such that for every $0<\delta<\varepsilon$, the fattening $D_{\delta}$ is simple.

Definition 6.6. We will say that a contractible vh-domain $R$ in $X$ or $Z$ is a generalized rectangle if its oriented boundary shape is $l l(r l)^{j} l l(r l)^{k}$, 
$|j-k| \leq 1$, and every inside shadow of stair-like turning-points is obstructed. We will say that a real-valued function is linear on $R$ if it is continuous and linear on every trapezium determined by $R$.

Definition 6.7. A pseudo- $\mathrm{vh}_{\sigma}$-domain will be called elementary if there is an $\varepsilon>0$ such that for every $0<\delta<\varepsilon$, the fattening $D_{\delta}$ is elementary, and every island of $D$ is a generalized rectangle.

REMARK 6.8. If a $\mathrm{vh}_{\sigma^{-}}$-domain $D$ is not simple, then $D=E \cup D^{\prime}$, where $E$ is elementary, has only one island, which is a rectangle, and $D^{\prime}$ is a pseudo$\mathrm{vh}_{\sigma}$-domain, possibly without interior, and $\operatorname{ord}(D)-\operatorname{ord}\left(D^{\prime}\right) \in\{2,4\}$.

Definition 6.9. Let $K_{1} \leq K_{2}$ be $v_{\sigma}$-fibers and let $D=D\left(K_{1}, K_{2}\right)$. A pseudo-roof on $D$ consists of a pair of functions $f_{1}, f_{2}: D \rightarrow[0,1]$ and a finite linearly ordered set $F \subseteq \mathcal{V} h_{\sigma}(D)$, called the critical fibers of the pseudo-roof, containing $K_{1}$ and $K_{2}$ and satisfying the following conditions.

(a) $f_{1}$ takes the value 0 on $K_{1}$, and $f_{2}$ takes the value 1 on $K_{2}$.

(b) $f_{1}$ is lower semicontinuous, $f_{2}$ is upper semicontinuous, and $f_{1} \leq f_{2}$. The functions coincide and are continuous on the interior of $D$.

(c) For every $r \in[0,1]$, the set $F_{r}=\left\{p \mid f_{1}(p) \leq r \leq f_{2}(p)\right\}$ is a $\mathrm{vh}_{\sigma}$-fiber.

(d) If $f_{1}(p)<f_{2}(p)$, then $p$ belongs to at least two distinct critical fibers.

(e) There is a finite sequence $0=r_{0}<r_{1}<\cdots<r_{n}=1$ such that $F=\left\{F_{r_{0}}, F_{r_{1}}, \ldots, F_{r_{n}}\right\}$.

(f) For every pair of consecutive values $r_{i}, r_{i+1}$, the $\mathrm{vh}_{\sigma}$-domain $D_{i}=$ $D\left(F_{r_{i}}, F_{r_{i+1}}\right)$ is simple or elementary, and $f_{1}=f_{2}$ is linear in the interior of any trapezium or rectangle determined by the islands of the $D_{i}$ 's. Note that the restrictions of $f_{1}$ and $f_{2}$ to $D_{i}$ are determined by their values on the fibers $F_{r_{i}}$ and $F_{r_{i+1}}$, except in the case of an elementary domain whose island is the shadow of a stair-like turn. In this case there are two choices. The level curves may be either horizontal or vertical.

Definition 6.10. A vh-pseudo-roof $\left(f_{1}, f_{2}, F\right)$ on $D$ will be called orderreducing if $\operatorname{ord}\left(F_{i}\right)$ is non-increasing on an interval of the form $\{0, \ldots, l\}$, and non-decreasing on an interval of the form $\{l, \ldots, n\}$, where $0 \leq l \leq n$.

Definition 6.11. The distance between two vh-pseudo-roofs $\left(f_{1}, f_{2}, F\right)$, and $\left(f_{1}^{\prime}, f_{2}^{\prime}, F^{\prime}\right)$ is the number $\max \left\{d_{F}\left(F_{r}, F_{r}^{\prime}\right) \mid r \in[0,1]\right\}$. We define the distance between regular roofs and vh-pseudo-roofs in exactly the same way.

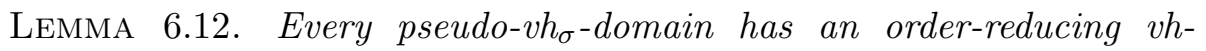
pseudo-roof, and for every vh-pseudo-roof $\left(f_{1}, f_{2}, F\right)$ on $D$, there is a domain $D^{\prime} \supseteq D$ and a vh-roof $f^{\prime}$ on $D^{\prime}$, arbitrarily close, which can be chosen to be order-reducing if $\left(f_{1}, f_{2}, F\right)$ is. 
Proof. If $D$ is simple, we leave it to the reader to see that we have a unique reducing vh-pseudo-roof, and for all sufficiently small $\delta>0$, the vh-roof on the fattening $D_{\delta}$ is a vh-roof of distance less than $\delta$. Let $D$ be a pseudo-vh ${ }_{\sigma}$-domain which is not simple. We may assume that $D=D^{\prime} \cup E$, where $E$ is elementary and the left boundary of $E$ coincides with the right boundary of $D^{\prime}$, and that this boundary has order strictly less than the order of the right boundary of $D$ or $E$. By induction we have an orderreducing vh-pseudo-roof $\left(f_{1}^{\prime}, f_{2}^{\prime}, F^{\prime}\right)$ on $D^{\prime}$ and a vh-pseudo-roof $\left(f_{1}^{\prime \prime}, f_{2}^{\prime \prime}, F^{\prime \prime}\right)$ on $E$. Then for any $k>0$ the triple $\left(f_{1}, f_{2}, F\right)$ defined by $F=F^{\prime} \cup F^{\prime \prime}$ and

$$
\begin{aligned}
& f_{1}(x)= \begin{cases}\frac{f_{1}^{\prime}(x)+k}{1+k} & \text { for } x \in D^{\prime}, \\
\frac{k \cdot f_{1}^{\prime \prime}(x)}{1+k} & \text { for } x \in E \backslash D^{\prime},\end{cases} \\
& f_{2}(x)= \begin{cases}\frac{f_{2}^{\prime}(x)+k}{1+k} & \text { for } x \in D^{\prime} \backslash E, \\
\frac{k \cdot f_{2}^{\prime \prime}(x)}{1+k} & \text { for } x \in E,\end{cases}
\end{aligned}
$$

is an order-reducing vh-pseudo-roof on $D$.

To approximate a vh-pseudo-roof $\left(f_{1}, f_{2}, F\right)$ on $D$, let $\delta>0$. Let $\operatorname{grid}(F)$ be the grid consisting of the extensions of all legs of fibers in $F$, and let $\varepsilon>0$ be so small that the fattening $\operatorname{grid}(F)_{\varepsilon}$ is homotopy equivalent to $\operatorname{grid}(F)$. We suppose that $\delta<\varepsilon$. Suppose $F=\left\{F_{r_{0}}, F_{r_{1}}, \ldots, F_{r_{n}}\right\}$. Let $f_{i}:\left(F_{r_{i}}\right)_{\delta} \rightarrow[0,1]$ be the vh-roof on the $\delta$-fattening of the $i$ th critical fiber, and let $K_{i}$ be the fiber $f_{i}^{-1}(i / n)$. The domains $D_{i}=D\left(K_{i}, K_{i+1}\right)$ are simple or elementary $\mathrm{vh}_{\sigma}$-domains. We choose a vh-roof $g_{i}$ on $D_{i}$. If $D_{i}$ is simple there is only one choice, and if $D_{i}$ is elementary we choose $g_{i}$ so that the extra triangles can be contained in a rectangle of thickness $\delta / n$. In the case where the elementary domain is of the type corresponding to a stair-like turn, we have to make sure that we choose the triangle so that the level-curves of $g_{i}$ are parallel to the level-curves of $f_{1}$ or $f_{2}$ outside the triangle. Then if we define $g$ so that

$$
\left.g\right|_{D_{i}}=\left(r_{i+1}-r_{i}\right) g_{i}+r_{i}
$$

we obtain $d\left(g,\left(f_{1}, f_{2}, F\right)\right) \leq \delta$.

Before we end this section we will introduce the notion of elementary distortions, and leftmost and rightmost fibers of a pseudo-vh $\sigma$-domain, and their properties.

Definition 6.13. A leg $a$ of $\mathrm{a}_{\sigma}$-fiber $F$ in a pseudo-vh $\sigma^{\text {-domain }} D$ will be called right movable if the following holds: 
(i) There are $\operatorname{vh}_{\sigma}$-fibers $F^{\prime}$ in $D$, with $F^{\prime}<F$, not meeting the interior of the leg $a$, and such that the interior of the pseudo-domain $D\left(F^{\prime}, F\right)$ is a single rectangle whose boundary has at most two legs contained in $F^{\prime}$.

(ii) The fibers $F^{\prime}$ satisfying (i) have a minimum.

If a leg $a$ is right movable and $F^{\prime}$ is a fiber satisfying (i), we say that $F^{\prime}$ is a right distortion of $F$ at $a$. The minimum fiber satisfying (i) is called the maximum right distortion of $F$ at $a$. If $F^{\prime \prime}$ is the maximum right distortion of $F$ at $a$ and $\operatorname{ord}\left(F^{\prime \prime}\right)<\operatorname{ord}(F)$, we call any right distortion of $F$ at $a$ strict. If $\operatorname{ord}\left(F^{\prime \prime}\right)=\operatorname{ord}(F)$, we call any right distortion of $F$ at a neutral. We define left movability and left distortions similarly. We say a leg is movable if it is either left or right movable.
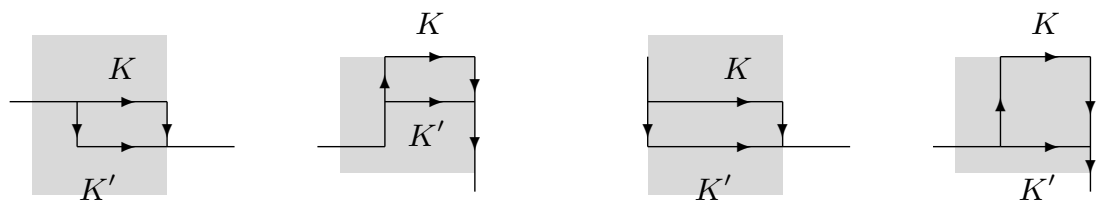

Fig. 5. In the first figure $K^{\prime}$ is a neutral right distortion of $K$ and $K$ is a maximal neutral left distortion of $K^{\prime}$. The next three figures show proper right distortions, the last two are maximal.

Definition 6.14. For a vh ${ }_{\sigma}$-fiber $F$ in a pseudo-vh $\sigma^{\text {-domain }} D$ we have the following sets that may not be disjoint:

Lmove $_{s}(F, D)=$ the set of strictly left movable legs of $F$ in $D$,

Rmove $_{s}(F, D)=$ the set of strictly right movable legs of $F$ in $D$,

Lmove $_{n}(F, D)=$ the set of neutrally left movable legs of $F$ in $D$,

Rmove $_{n}(F, D)=$ the set of neutrally right movable legs of $F$ in $D$.

Definition 6.15. For vh ${ }_{\sigma}$-fibers $F<F^{\prime}$ in a pseudo-vh ${ }_{\sigma}$-domain $D$, we call $F^{\prime}$ a simple left distortion of $F$ if there is a left movable leg $a$ of $F$ such that $F^{\prime}$ is a left distortion of $F$ at $a$. We call $F^{\prime}$ a semisimple left distortion of $F^{\prime}$ if there is a sequence $F_{0}<F_{1}<\cdots<F_{m}$ such that $F_{0}=F, F_{m}=F^{\prime}$, $F_{i+1}$ is a simple left distortion of $F_{i}$ for $0 \leq i \leq m-1$, and the interior of the domain $D\left(F, F^{\prime}\right)$ consists of mutually disjoint open generalized rectangles. If $\mathrm{P}$ is one of the properties: right, left, maximum, strict, neutral, we call a semisimple distortion $\mathrm{P}$ if it is possible to choose a sequence of intermediate simple distortions which are all $\mathrm{P}$.

REMARK 6.16. Note that the intermediate simple distortions are not unique, but the generalized rectangles are, so the definition does not depend on the particular sequence of simple distortions. 
Definition 6.17. A $v_{\sigma}$-fiber $F$ in a pseudo-vh $\sigma^{\text {-domain }} D$ will be called rightmost if none of the legs of $F$ are right movable, which means that $\operatorname{Rmove}_{s}(F, D)=\operatorname{Rmove}_{n}(F, D)=\emptyset$. Similarly we define a leftmost fiber.

Note that a fiber of order zero is both leftmost and rightmost. In general the closest we can get to fibers that are both leftmost and rightmost is the following.

Definition 6.18. A $\operatorname{vh}_{\sigma}$-fiber $F$ in a pseudo-vh $\sigma^{\text {-domain }} D$ will be called central if it has no strictly movable legs and at most one neutrally movable leg. This means that $\operatorname{Rmove}_{n}(F, D) \cap \operatorname{Lmove}_{n}(F, D) \in\{\emptyset,\{a\}\}$. If $F$ is central and has a two-way movable leg $a$, we call it the two-way movable leg of $F$. If we move all the right movable legs of a central fiber $F$ all the way to the right, we get a fiber $F_{1}$ of the same order as $F$, and $F_{1}$ is a right neutral semisimple distortion of $F$. Similarly we get a left neutral semisimple distortion $F_{2}$ of $F$, and $F_{1}$ and $F_{2}$ are neutral semisimple distortions of each other. A subdomain of a pseudo-domain $D$ of the form $D\left(F_{1}, F_{2}\right)$, where $F_{1}$ is the maximal right distortion and $F_{2}$ the maximal left distortion of a central fiber $F$, will be called a central domain of $D$.

Proposition 6.19. Let $D=D\left(F_{1}, F_{2}\right)$ be a pseudo-vh ${ }_{\sigma}$-domain such that $F_{1}$ has no strict left movable leg, and $F_{2}$ has no strict right movable leg. Then $F_{1}$ and $F_{2}$ are maximal neutral semisimple distortions of each other.

Proof. Let $R$ be the closure of a connected component of $D$. By our assumption the part of the boundary that meets $F_{1}$ cannot have two consecutive left turning-points, and the part of the boundary that meets $F_{2}$ cannot have two consecutive right turning-points. This shows that $R$ is contractible. When we move around the boundary of $R$ counterclockwise we must have a surplus of four l's and therefore at least four circular occurrences of $l l$. Also every shadow of a right stair-like turning-point of $F_{2}$ must be obstructed by $F_{1}$ and vice versa. It follows that the shape of $R$ is $l l(r l)^{k} l l(r l)^{k}$ or $l l(r l)^{k} l l(r l)^{k-1}$ for some $k \geq 1$. This shows the proposition.

Definition 6.20. A sequence of $\operatorname{vh}_{\sigma}$-fibers $K_{1}=F_{0}<F_{1}<\cdots$ $<F_{l+m}=K_{2}$ will be called special if the following holds:

(i) For $i \in\{0, l-1\}, F_{i+1}$ is a semisimple maximal left strict distortion of $F_{i}$.

(ii) The fibers $F_{l}$ and $F_{l+1}$ are maximal neutral semisimple distortions of each other.

(iii) For $i \in\{l, l+m-1\}, F_{i}$ is a maximal right strict semisimple distortion of $F_{i+1}$. 
Definition 6.21. A vh-pseudo-roof $f_{1}, f_{2}: D \rightarrow[0,1]$ on $D=D\left(K_{1}, K_{2}\right)$ will be called special if its critical fibers $K_{1}=F_{0}<F_{r_{1}}<\cdots<F_{r_{n}}=F_{1}=K_{2}$ form a special sequence.

Note that special sequences are not unique, but the length might be well defined if every distortion is simple. I do not know the answer.

Corollary 6.22. Every pseudo-vh $h$-domain $D\left(K_{1}, K_{2}\right)$ has a special roof.

The pseudo-vh ${ }_{\sigma}$-domains that will play a leading role in what follows are the ones that we get by filling in the closure of the shadows of all or some of the unobstructed tags and double tags of a $\mathrm{vh}_{\sigma}$-curve. Such pseudo-domains allow a decomposition into directed rectangles and hooks, i.e. domains of shape lllllr. See Figure 6.

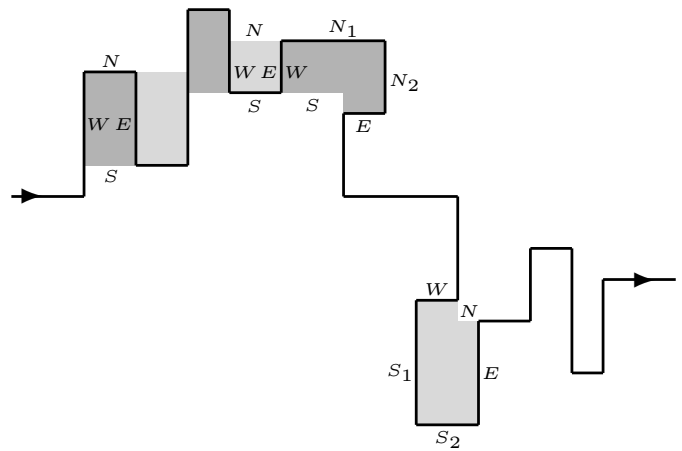

Fig. 6. Part of a directed pseudo-domain. There are two right rectangles, two left rectangles, one right hook and one left hook. The darker ones are the right ones.

Definition 6.23. A direction on a rectangle $R$ in $X$ or $Z$ is a naming of its sides in positive order, east, north, west, south, together with a value right or left. We write east $(R)$ for the eastern edge of $R$, and similarly for the other directions. We will also consider degenerate directed rectangles. A degenerate directed rectangle is simply a line-segment with an eastern end a western end. The degenerate rectangles do not have a value right or left, and their only leg is both a northern and a southern edge. Even though their eastern and western edges are just points we will consider them to have direction perpendicular to the long edge. The reader may feel free to think non-standard.

Definition 6.24. A direction on a hook $L$ in $X$ or $Z$ is a naming of its sides in positive order, east, north 2 , north 1 , west, $\operatorname{south}_{1}$, south $_{2}$, together with a value right or left. The value of a directed hook is actually determined by the naming of the edges. The value left is given to the hooks for which the right turn is between the northern edges, and they will be called left-hooks. 
The right-hooks have the right-turn between the southern edges. We write east $(L)$ for the eastern edge of $L$, and similarly for the other directions.

Definition 6.25. A directional decomposition of a pseudo- $\mathrm{vh}_{\sigma}$-domain $D=D\left(K_{1}, K_{2}\right)$ consists of a finite set $\mathcal{S}$ of directed rectangles and hooks, $\mathcal{S}=\mathcal{S}_{L R} \cup \mathcal{S}_{R R} \cup \mathcal{S}_{L H} \cup \mathcal{S}_{R H} \cup \mathcal{S}_{\mathrm{deg}}$, such that:

(i) $D=\bigcup \mathcal{S}$.

(ii) There is a circular enumeration $S=\left\{S_{0}, S_{1}, \ldots, S_{n}\right\}$ such that only neighbors meet, and $S_{j} \cap S_{j+1} \subseteq \operatorname{east}\left(S_{j}\right) \cap \operatorname{west}\left(S_{j+1}\right)$.

(iii) For each $S_{j} \in \mathcal{S}, \operatorname{south}\left(S_{j}\right) \subseteq K_{1}$ and $\operatorname{north}\left(S_{j}\right) \subseteq K_{2}$.

(iv) If two non-degenerate members of $\mathcal{S}$ touch, they touch along parallel east-west edges and have opposite values.

(v) The northern edge or edges of a left rectangle or hook always extend to the northern edge to at least one of its neighbors. Ditto for the southern edge or edges of right rectangles or hooks.

We will say that a directed pseudo-domain $(D, \mathcal{S})$ is eligible if $\mathcal{S}$ contains both left and right elements.

If $\mathcal{S}$ is a directional decomposition of a pseudo- $\mathrm{vh}_{\sigma}$-domain $D=$ $D\left(K_{1}, K_{2}\right)$, we can single out a special subspace of $\mathcal{V} h_{\sigma}(D)$ consisting of $\mathcal{S}$-fibers, and we also define a special pseudo-metric $d_{\mathcal{S}}$ on the $\mathcal{S}$-fibers.

Definition 6.26. The $\mathcal{S}$-fibers $K \in \mathcal{V} h_{\sigma}(D)$ are the fibers with no turning-points in the interior of any of the rectangles of $\mathcal{S}$, and exactly one turning-point in every hook of $\mathcal{S}$. We denote the set of $\mathcal{S}$-fibers by $\mathcal{T}(D, \mathcal{S})$.

Definition 6.27. For a member $S \in \mathcal{S}$ and $\mathcal{S}$-fibers $K_{1}$ and $K_{2}$, we define $d_{S}^{(-)}\left(K_{1}, K_{2}\right)$ to be the distance between $K_{1}$ and $K_{2}$ immediately after entering $S$, and $d_{S}^{(+)}\left(K_{1}, K_{2}\right)$ to be the distance between $K_{1}$ and $K_{2}$ immediately before leaving $S$. We define $d_{S}=d_{S}^{(-)}+d_{S}^{(+)}$, and finally

$$
d_{\mathcal{S}}=\sum_{S \in \mathcal{S}} d_{S}
$$

Note that $d_{\mathcal{S}}$ is a metric when restricted to $\mathcal{S}$-fibers.

Definition 6.28. An $\mathcal{S}$-vh-pseudo-roof on $(D, \mathcal{S})$ is a vh-pseudo-roof on $D$ whose fibers are $\mathcal{S}$-fibers.

Lemma 6.29. The $\mathcal{S}$-fibers enjoy the following properties.

(i) The space $\mathcal{T}(D, \mathcal{S})$ is a lattice, but not a sublattice of $\mathcal{V} h_{\sigma}(D)$.

(ii) The order-function is lower semicontinuous.

(iii) If $K_{1} \leq L \leq K_{2}$, then $d_{\mathcal{S}}\left(K_{1}, L\right)+d_{\mathcal{S}}\left(K_{2}, L\right)=d_{\mathcal{S}}\left(K_{1}, K_{2}\right)$.

(iv) $d_{\mathcal{S}}\left(K_{1}, K_{2}\right)=d_{\mathcal{S}}\left(K_{1} \wedge K_{2}, K_{1} \vee K_{2}\right)$.

Proof. Left to the reader. 
It follows from Lemma 6.29(ii) that the order-function has a minimum value.

Definition 6.30. The order of a directional decomposition $\mathcal{S}$, $\operatorname{ord}(\mathcal{S})$, on a $\operatorname{vh}_{\sigma}$-domain $(D, \mathcal{S})$ is the maximum value the order of an $\mathcal{S}$-fiber.

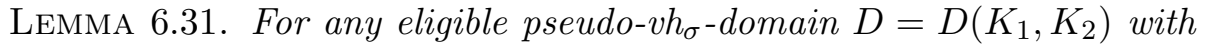
directional decomposition $\mathcal{S}$, we have $\operatorname{ord}(D)<\operatorname{ord}(\mathcal{S})$.

Proof. This is because by Definition $6.25(\mathrm{v}), K_{1}$ will skip a corner along the southern edge of a right rectangle or hook, and $K_{2}$ will skip a corner along the northern edge of a left rectangle or hook, and since it is eligible, there are both left and right elements of $\mathcal{S}$.

EXAMPLE 6.32 . Let $K$ be a $\operatorname{vh}_{\sigma}$-fiber with a $v_{\sigma}$-curve $\gamma=\gamma(K)$. Let $D$ be the $\mathrm{vh}_{\sigma}$-domain obtained by taking the union of $K$ and the closures of some of the unobstructed tags of $\gamma$. Then if we have added the shadows of both some left tags and some right tags, we get a $\mathrm{vh}_{\sigma}$-domain with a directional decomposition $\mathcal{S}$ consisting of the closures of the shadows of the simple tags as the left and right rectangles, and the closures of the shadows of the twin tags as the hooks. The rest will be the degenerate rectangles. This decomposition is unique as long as we do not split legs into more than one degenerate rectangle. Note that the right tags correspond to right rectangles.

Note that there will be many different directed $\mathrm{vh}_{\sigma}$-domains corresponding to a $v_{\sigma}$-fiber $K$. If we fill in the closures of the shadows of all the unobstructed tags of $K$, we get the maximal directed $\mathrm{vh}_{\sigma}$-domain corresponding to $K$, and this is always eligible by Lemma 3.32 .

Definition 6.33. The maximal directed $\mathrm{vh}_{\sigma}$-domain corresponding to a $\operatorname{vh}_{\sigma}$-fiber $K$ of positive order will be denoted by $(D(K), \mathcal{S}(K))$.

Lemma 6.34. For any directed $v h_{\sigma}$-domain $(D, \mathcal{S})$ such that no parallel degenerate edges are joined, there is a unique vh ${ }_{\sigma}$-fiber $K$ with $D \subseteq D(K)$, $\mathcal{S} \backslash \mathcal{S}_{\mathrm{deg}} \subseteq \mathcal{S}(K) \backslash \mathcal{S}(K)_{\mathrm{deg}}$, and $\operatorname{ord}(K)=\operatorname{ord}((D, \mathcal{S}))$.

Proof. Let

$$
\partial^{\prime}(S)= \begin{cases}\bigcup\{\text { west }(S), \operatorname{south}(S), \operatorname{east}(S)\} & \text { for } S \in \mathcal{S}_{L R}, \\ \bigcup\{\operatorname{west}(S), \operatorname{north}(S), \operatorname{east}(S)\} & \text { for } S \in \mathcal{S}_{R R}, \\ \bigcup\left\{\operatorname{west}(S), \operatorname{south}_{1}(S), \operatorname{south}_{2}(S), \operatorname{east}(S)\right\} & \text { for } S \in \mathcal{S}_{L H}, \\ \bigcup\left\{\operatorname{west}(S), \operatorname{north}_{1}(S), \operatorname{north}_{2}(S), \operatorname{east}(S)\right\} & \text { for } S \in \mathcal{S}_{R H}, \\ S & \text { for } S \in \mathcal{S}_{\text {deg }} .\end{cases}
$$

Then $K=\bigcup\left\{\partial^{\prime}(S) \mid S \in \mathcal{S}\right\}$. 
Before we end this section we will prove some properties of leftmost, rightmost and central $\mathcal{S}$-fibers of a directed pseudo-vh ${ }_{\sigma}$-domain.

Definition 6.35. An $\mathcal{S}$-fiber $F$ in a directed $\operatorname{vh}_{\sigma}$-domain $(D, \mathcal{S})$ will be called a central $\mathcal{S}$-fiber if either $F$ is central, or there are central fibers $F_{1}<F<F_{2}$ without two-way movable legs and $F$ contains consecutive legs $c, a, d, \operatorname{Rmove}_{s}(F)=\{a, c\}, \operatorname{Lmove}_{s}(F)=\{a, d\}$ such that $F_{1}$ is a strict right simple distortion of $F$ both at $a$ and $c$, and $F_{2}$ is a strict left simple distortion of $F$ both at $a$ and $d$.

Definition 6.36. By an extreme $\mathcal{S}$-fiber we will mean an $\mathcal{S}$-fiber that is either leftmost, rightmost or central.

Definition 6.37. Let $(D, \mathcal{S})$ be a directed pseudo-domain, and $E$ a set of east-west edges along which two consecutive members of $\mathcal{S}$ are attached. We denote by $\mathcal{T}^{E}(D, \mathcal{S})$ the space of $\mathcal{S}$-fibers that cut straight through the borders in $E$.

Lemma 6.38. In a directed vh ${ }_{\sigma}$-domain, $(D(K), \mathcal{S}(K))=D\left(K_{1}, K_{2}\right)$, the following holds:

(i) An $\mathcal{S}$-fiber $M$ is a rightmost (resp. leftmost) $\mathcal{S}$-fiber if and only if every leg of $M$ of the form $\mathrm{rr}, \mathrm{rl}$ or $\mathrm{lr}$ (resp. ll, lr or $r l$ ) contains a leg of $K_{1}$ (resp. $\left.K_{2}\right)$. In particular, every north-south leg of $M$ contains a leg of $K_{1}$ (resp. $\left.K_{2}\right)$.

(ii) No leftmost or rightmost $\mathcal{S}$-fiber enters or leaves a right member of $\mathcal{S}$ at a left turning-point, nor does it enter or leave a left member of $\mathcal{S}$ at a right turning-point.

(iii) If $M$ is rightmost and cuts straight through an east-west attaching border, then all rightmost or central $\mathcal{S}$-fibers $M^{\prime}$ with $M \leq M^{\prime}$ in the neighboring rectangles of this border cut straight through the same border.

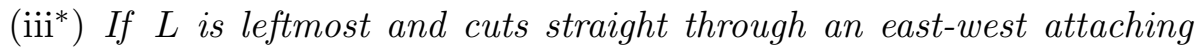
border, then all leftmost or central $\mathcal{S}$-fibers $L^{\prime}$ with $L^{\prime} \leq L$ in the neighboring rectangles of this border cut straight through the same border.

(iv) If $M$ and $L$, then except if $L$ is leftmost and $M$ is rightmost and $M<L$, there is an east-west border e along which two consecutive members of $\mathcal{S}$ are attached, such that both $M$ and $L$ are members of $\mathcal{T}^{\{e\}}(D, \mathcal{S})$.

Proof. We leave the proof of (i) and (ii) to the reader. We proceed to prove (iii). If $M^{\prime}$ has a leg along this border, it could be moved in a neutral, improper way to make a right distortion, contradicting the fact that $M^{\prime}$ is rightmost. This also shows that (iv) holds in case the fibers are both central, 
one central and one rightmost or leftmost, both rightmost or both leftmost, and in the case of comparable leftmost and rightmost fibers. It remains to check the case of non-comparable rightmost and leftmost fibers. Assume then that $L$ is leftmost, $M$ is rightmost and $L \not Z M$ and $M \not L L$. Let $p$ be a left turning-point of $L$ on the right side of $M$. Since the north-south leg adjacent to $q$ meets $K_{2}$, which is the very leftmost fiber of $D, M$ has to cross or merge with this leg between $q$ and where the leg meets $K_{2}$. Since $L$ is leftmost, and $M$ is to the left of $L$ at $p$, the turn $p$ preceding $q$ is a right turning-point. By (i) this forces the leg $p, q$ to meet $K_{2}$ as well, and therefore $M$ crosses or merges with the leg $p, q$ between $q$ and where the leg meets $K_{2}$. By symmetry, $M$ has a right turning-point $s$ on the right side of $L$ between the points where it meets $L$ on either side of $q$, and the legs of $M$ adjacent to $s$ both meet $K_{1}$. This shows that the square determined by the points $q$ and $s$ meet an even number of rectangles from $\mathcal{S}$, and this proves (iv).

7. Moving directed pseudo-vh $\mathbf{h}_{\sigma}$-domains. In this section we study families of $\mathrm{vh}_{\sigma}$-fibers $K_{t}$, and directed pseudo-domains $\left(D_{t}, \mathcal{S}_{t}\right)$ contained in $\left(D\left(K_{t}\right), \mathcal{S}\left(K_{t}\right)\right)$. The problem is that even if $K_{t}$ is continuous, $\left(D\left(K_{t}\right), \mathcal{S}\left(K_{t}\right)\right)$ might not be, even if the shape of $K_{t}$ stays constant. If the family has constant order, the shape will also stay constant, and we need to keep track of the tags of $K_{t}$.

Definition 7.1. Let $w \in\{l, r\}^{2 n}$, and let $K$ be a fiber with shape $[w]$. A matching $m$ for the fiber $K$ in $w$ is an order-preserving correspondence $m$ between the turning-points of $K$ and the word $w$.

Note that if $|w|=2 n$, the number of such matchings equals the order of the isotropy subgroup of $w$ in the translation group $\mathbb{Z} / 2 n \mathbb{Z}$ acting on $\{l, r\}^{2 n}$. In particular, there are exactly two matchings in the case $w=$ rrllrrll.

Definition 7.2. For a matched fiber $(K, m, w)$ we have a subset which we name $\operatorname{Unobst}(K, m, w) \subseteq \operatorname{circocc}(r r, w) \cup \operatorname{circocc}(l l, w)$, consisting of the circular occurrences that correspond to unobstructed tags.

LEMMA 7.3. Let $U$ be a connected component of $\mathcal{V} h_{\sigma}^{(2 n)} \backslash \mathcal{V} h_{\sigma}^{(2 n-2)}$ of shape $[w]$. Let $U^{*}$ be the cover on which $m$ is globally well defined. Then the function Unobst : $U^{*} \rightarrow P(\operatorname{circocc}(r r, w) \cup \operatorname{circocc}(l l, w))$ is lower semicontinuous. In other words, for any $V \subseteq \operatorname{circocc}(r r, w) \cup \operatorname{circocc}(l l, w)$, the set $\{(K, m) \mid V \subseteq \operatorname{Unobst}(K, m)\}$ is open.

Proof. If a tag of a matched fiber $(K, m)$ is unobstructed, the legs not adjacent to the tag have some distance from the tag, so the tag remains unobstructed in a neighborhood. Obstructed tags, on the other hand, may be unobstructed at fibers arbitrarily close. 
Note that Unobst is constant in the case $w=$ rrllrrll and may vary with subsets having from two to five members in the case $w=$ rllrllrrrrl. By Lemma 3.32, the cardinality of any subset $\operatorname{Unobst}(K, m)$ is at least 2 .

Definition 7.4. Let $w \in\{l, r\}^{2 n}$, and let $E \subseteq \operatorname{circocc}(r r, w) \cup$ $\operatorname{circocc}(l l, w)$. If $K$ is a fiber with shape $[w]$ and $m$ is a matching for $K$ in $w$, we write $\mathcal{S}_{E}(K)=\mathcal{S}_{E}(K, m, w)$ for the subset of $\mathcal{S}(K)$ consisting of the rectangles and hooks corresponding, through $m$, to occurrences of $x x$ 's, or of $x x x^{\prime}$ s such that both suboccurrences of $x x$ belong to $E$. We write $D_{E}(K)=D_{E}(K, m, w)$ for the corresponding directed pseudo-domain. We will call $E$ eligible for $(K, m, w)$ if $\left(D_{E}(K, m, w), \mathcal{S}_{E}(K, m, w)\right)$ is eligible (see Definition 6.25).

Definition 7.5. Let $K_{t}, t \in\langle a, b\rangle$, be a continuous family of fibers in $\mathcal{V} h_{\sigma}^{(2 n)} \backslash \mathcal{V} h_{\sigma}^{(2 n-2)}$ of shape $[w]$, and let $m_{t}$ be a continuous matching. A subset $E \subseteq \operatorname{circocc}(r r, w) \cup \operatorname{circocc}(l l, w)$ will be called stable at a point $t_{0} \in\langle a, b\rangle$ if there is a neighborhood $U$ of $t_{0}$ such that $D_{E}\left(K_{t}, m_{t}, w\right)$ is continuous (in the Hausdorff metric).

Note that $E$ is stable at $t_{0}$ if and only if $\operatorname{Unobst}\left(K_{t}, m_{t}, w\right) \cap E$ is constant for $t$ near $t_{0}$. From Lemma 7.3, we get the following corollary.

Corollary 7.6. For any continuous matched family $\left(K_{t}, m_{t}, w\right)$ and any interior point $t_{0}$, the set $E=\operatorname{Unobst}\left(K_{t_{0}}, m_{t_{0}}, w\right)$ is eligible and stable.

The main result of this section is the following.

Proposition 7.7. Let $K_{t}, t \in[0,1\rangle$, be a continuous family of fibers with $\operatorname{ord}\left(K_{t}\right)=2 n$ for $t>0$. Assume that $w$ and $v$ are the shapes of $K_{t}$ and $K_{0}$, and that $m_{t}$ and $n$ are matchings such that we can write $v=\alpha_{1}, \ldots, \alpha_{2 k}$, $w=x_{1}, y_{1}, \ldots, x_{2 k}, y_{2 k}$ and for a turning-point $p(t)$, the letter $m_{t}(p(t))$ occurs in $x_{i}$ if and only if $\lim p(t)$ is a turning-point of $K_{0}$ and $n(\lim p(t))$ $=\alpha_{i}$, and it occurs in $y_{i}$ if and only if $\lim p(t) \in\left\langle n^{-1}\left(\alpha_{i}\right), n^{-1}\left(\alpha_{i+1}\right)\right\rangle$. Let $E$ be the set of $x x$ occurrences in $w$ occurring in the words $x_{i} y_{i} x_{i+1}$ for which $\alpha_{i}, \alpha_{i+1}$ correspond to an unobstructed tag in $K_{0}$. Then the following holds:

(i) The limit $\lim _{t \rightarrow 0^{+}} D_{E}\left(K_{t}\right)=D_{0}$ exists, and is contained in $D\left(K_{0}\right)$.

(ii) There is an $\varepsilon>0$ such that $E$ is eligible for all $0<t<\varepsilon$.

Proof. We see (i) because the rectangles and hooks of $\mathcal{S}_{E}\left(K_{t}, m_{t}, w\right)$ that do not persist get absorbed by the interiors of the legs of $K_{0}$ as $t$ tends to zero. Next let $\varepsilon$ be so small that all vanishing turning-points $p(t)$ converge to points in closed intervals $\left[q-\varepsilon, q^{\prime}+\varepsilon\right]$ of legs $\left[q, q^{\prime}\right]$ of $K_{0}$, and $\operatorname{Unobst}\left(K^{\prime}, n^{\prime}, v\right)=\operatorname{Unobst}\left(K_{0}, n, v\right)$ for all matched fibers $\left(K^{\prime}, n^{\prime}, v\right)$ of distance less than $\varepsilon$ from $\left(K_{0}, n, v\right)$. We consider a rectangular domain 
of thickness less than $\varepsilon$ containing the middle leg of an unobstructed tag, hosting tag, of $K_{0}$. For $t<\varepsilon$ we join the part of $K_{t}$ in this rectangle to a leg parallel to the middle leg of the hosting tag. This will form a right (resp. left)-turning closed curve if the hosting tag is a right (resp. left) tag, and the proposition follows from Lemmas 3.31 and 3.32 .

Note that the limit $D_{0}$ may not be eligible. It can in fact be $K_{0}$ itself.

8. Reduction. In this section we prove a key result. As in the earlier sections, we consider the spaces $Y, Z, X$, with $\sigma$-coordinates $x$ and $\widetilde{x}$. We also fix an intrinsic simple topological measure $\mu$ on $X$. From now on we simplify our language and drop the index $\sigma$. The $\mathrm{vh}_{\sigma}$-fibers are simply called fibers, and fibers with $\mu$-measure equal to 1 are called $\mu$-fibers. Before we prove the key lemma we make some simple observations.

LEMma 8.1. If $\Upsilon$ is any bounded space of fibers in a pseudo-domain $D$, say in the Hausdorff metric, the subspace $\Upsilon_{\mu}$ of all $\mu$-fibers in $\Upsilon$ is closed in $\Upsilon$.

Proof. This follows from Definition 0.1 since the fibers are closed and $\Upsilon$ is a Hausdorff space.

Lemma 8.2. Let $F_{1} \leq F \leq F_{2}$ be fibers in a pseudo-domain $D$. Then if both $F_{1}$ and $F_{2}$ are $\mu$-fibers, so is $F$.

Proof. Let $K$ be a fiber in the complement $U$ of the (closed) pseudodomain $D\left(F_{1}, F_{2}\right)$. The interior $V_{1}$ of $D(F, K)$ has $\mu$-measure 0 because it is disjoint from $F_{1}$, and the interior $V_{2}$ of $D(K, F)$ has $\mu$-measure 0 because it is disjoint from $F_{2}$. By additivity $\mu\left(V_{1} \cup K \cup V_{2}\right)=0$, so $\mu(F)=1$.

Lemma 8.3. Let $f_{1}, f_{2}: D \rightarrow[0,1]$ be a pseudo-roof on a pseudodomain $D$. Then if $\mu(D)=1$, there is a non-empty interval $\left[t_{1}, t_{2}\right] \subseteq[0,1]$ such that $\mu\left(F_{t}\right)=1$ if and only if $t \in\left[t_{1}, t_{2}\right]$.

We consider a left movable leg $a$, and a right movable leg $b \neq a$ of a fiber $F$ in a pseudo-domain $D$. We let $F_{2}$ be the maximum simple left distortion of $F$ at $a$, and $F_{1}$ be the maximum simple right distortion of $F$ at $b$. We denote by $R_{1}$ the closed rectangle determined by $F$ and $F_{1}$, and we denote by $b^{\prime}$ the leg of $F_{1}$ opposite $b$. Similarly we have $F_{2}, R_{2}$ and $a^{\prime}$. We divide this situation into three distinct, exhaustive cases:

CASE 1: The rectangles are disjoint.

CASE 2a: The rectangles touch, and $a$ and $b$ are not parallel.

CASE 2b: The rectangles touch, and $a$ and $b$ are parallel.

Note that in Case 2a, $D\left(F_{1}, F_{2}\right)=R_{1} \cup R_{2}$ is almost always a hook, but possibly just one rectangle. In Case $2 \mathrm{~b}, R_{1} \cup R_{2}$ is almost always a shape 
of type $(l l r l)^{2}$, and in special cases a hook, a rectangle, or two rectangles meeting at a corner.

Definition 8.4. Let $D, F, a, a^{\prime}, b^{\prime}, b$ and $R_{1}, R_{2}$ be as above. A pair of line segments $a^{\prime \prime}$ and $b^{\prime \prime}$ in the rectangles $R_{1}$ and $R_{2}$ will be called admissible if either $a$ and $b$ are not parallel, or the smaller rectangles $R_{1}^{\prime \prime} \subseteq R_{1}$ determined by $a$ and $a^{\prime \prime}$ and $R_{2}^{\prime \prime} \subseteq R_{2}$ determined by $b^{\prime \prime}$ are either disjoint or meet only at a corner where necessarily $a^{\prime \prime}$ and $b^{\prime \prime}$ extend each other. We call the corresponding pair $\left(F_{1}^{\prime \prime}, F_{2}^{\prime \prime}\right)$ an admissible pair of simple distortions at the pair $(a, b)$. The fiber $F^{\prime \prime}$ with $\operatorname{ord}\left(F^{\prime \prime}\right) \leq \operatorname{ord}(F)$ of $D^{\prime \prime}=D\left(F_{1}^{\prime \prime}, F_{2}^{\prime \prime}\right)$ will be called the critical $\left(a^{\prime \prime}, b^{\prime \prime}\right)$-fiber of $F$ at $(a, b)$; it contains at least a part of $a^{\prime \prime}$ and $b^{\prime \prime}$. We denote by $\operatorname{Adm}(F, D, a, b)$ the space of all critical $\left(a^{\prime \prime}, b^{\prime \prime}\right)$-fibers of $F$ at $(a, b)$. A fiber $H \in \operatorname{Adm}(F, D, a, b)$ will be called maximal if either $a^{\prime}=a^{\prime \prime}, b^{\prime}=b^{\prime \prime}$, or the legs $a^{\prime \prime}$ and $b^{\prime \prime}$ are extensions of each other, in which case we are guaranteed that $\operatorname{ord}(H)<\operatorname{ord}(F)$.

Note that the critical fiber always divides the pseudo-domain $D^{\prime \prime}$ into rectangles.

Definition 8.5. Given $D, F, a, a^{\prime}, b^{\prime}, b$ and $R_{1}, R_{2}$ as above, and suppose that $F$ is a $\mu$-fiber. We will call a critical $(c, d)$-fiber $G \in \operatorname{Adm}(F, D, a, b)$ with $\mu(G)=1$ a $(c, d)$-reduction of $F$ at $(a, b)$. We denote by $\operatorname{Red}(F, D, a, b)$ the space of all $(c, d)$-reductions of $F$ at $(a, b)$. A $(c, d)$-reduction of $F$ at $(a, b)$ is maximal if it is maximal as a member of $\operatorname{Adm}(F, D, a, b)$.

The key lemma is this:

LEMMA 8.6. If $F$ is a $\mu$-fiber in a pseudo-domain $D$, and $(a, b)$ a pair of legs with a left movable and $b$ right movable, then the space $\operatorname{Red}(F, D, a, b)$ is connected and contains maximal reductions.

Proof. Note first that $\operatorname{Red}(G, D, c, d) \subseteq \operatorname{Red}(F, D, a, b)$ for any $(c, d)$ reduction $G$ of $F$ at $(a, b)$. Also if $G_{1}$ is a $\left(c_{1}, d_{1}\right)$-reduction with $d_{1}$ closest to $b^{\prime}$, and if $G_{2}$ is a $\left(c_{2}, d_{2}\right)$-reduction with $c_{2}$ closest to $a^{\prime}$, we have $G_{1} \leq G_{2}$, and so by Lemma 8.2 , there is a $\left(c_{2}, d_{1}\right)$-reduction of $F$ at $(a, b)$. Let $\left(a^{\prime \prime}, b^{\prime \prime}\right)$ be an admissible pair at $(a, b)$, and let $D^{\prime \prime}=D\left(F_{1}^{\prime \prime}, F_{2}^{\prime \prime}\right)$. Consider the reducing pseudo-roof $f_{1}, f_{2}: D^{\prime \prime} \rightarrow[0,1]$ with one critical fiber $F_{1 / 2}$ being the critical $\left(a^{\prime \prime}, b^{\prime \prime}\right)$-fiber at $F$ at $(a, b)$. Then every fiber $F_{t}$ either contains $b^{\prime \prime}$ or at least part of $a^{\prime \prime}$, so by Lemma 8.3 there is a fiber $G=F_{t}$ which is a $(c, d)$-reduction with $a^{\prime \prime}=c$ or $b^{\prime \prime}=d$. If $G$ is any $(c, d)$-reduction which is not maximal, then $c$ is a left movable and $d$ a right movable leg of $G$, and there is an admissible pair $\left(c^{\prime \prime}, d^{\prime \prime}\right)$ for $(c, d)$ and a $\left(c^{\prime}, d^{\prime}\right)$-reduction $G^{\prime}$ of $G$ which is also a $\left(c^{\prime}, d^{\prime}\right)$-reduction of $F$ and closer to a possible maximal one. This proves the lemma. 
Lemma 8.7. Let $F$ be a $\mu$-fiber in a pseudo-domain $D$ and suppose $F$ has legs $a \in \operatorname{Left}_{s}(F, D), b \in \operatorname{Right}_{s}(F, D)$ and $a \neq b$. If $F^{\prime}$ is a maximal $(a, b)$-reduction, then ord $F^{\prime}<$ ord $F$.

Definition 8.8. We let $\mathcal{V} h^{(2 n)}(D)$ be the space of fibers in a pseudodomain $D$ of order not exceeding $2 n$. The more interesting space is the subspace $\mathcal{V} h_{\mu}^{(2 n)}(D)$ of $\mu$-fibers in $\mathcal{V} h^{(2 n)}(D)$.

COROLlary 8.9. For every natural number n, every connected component of the compact space $\mathcal{V} h_{\mu}^{(2 n)}(D)$ contains either a leftmost, rightmost or central fiber.

Proof. For $n=0, \mathcal{V} h_{\mu}^{(2 n)}(D)$ is either empty or contains a single fiber, which is both leftmost, rightmost and central. For $n=1, \mathcal{V} h_{\mu}^{(2 n)}(D)$ is empty and the corollary holds trivially. Assume that the corollary does not hold and let $n$ be the smallest number for which there is a connected component $\mathcal{W}$ of $\mathcal{V} h_{\mu}^{(2 n)}(D)$ without a leftmost, rightmost or central fiber. By our choice of $n$, all fibers of $\mathcal{W}$ have order $2 n$, and do not contain leftmost, rightmost or central fibers. By Lemma 8.7, any fiber $F$ in $\mathcal{W}$ cannot have both left and right strictly movable legs. No fiber can have a single leg that is both left and right strictly movable, because a two-way strictly movable leg has strictly movable neighboring legs. Hence, by symmetry, we may assume that $\operatorname{Rmove}_{s}(F, D)=\emptyset$. If $\operatorname{Lmove}_{s}(F, D) \neq \emptyset$, it follows from Lemma 8.6 that we can use one of these legs to eliminate all right movable legs since by assumption the order cannot decrease. But then the result is a leftmost fiber. This is a contradiction, so $F$ has only neutrally movable fibers. Let $F$ be any fiber in $\mathcal{W}$ and assume that $\left|\operatorname{Lmove}_{n}(F, D) \cap \operatorname{Rmove}_{n}(F, D)\right|>1$. We can use the process of Lemma 8.6 to push all but possibly one of them to the extreme left or right. The result is a central fiber, and this contradicts our assumption.

Lemma 8.10. For any set $E$ of east-west edges of a directed pseudodomain $(D, \mathcal{S}), \mathcal{T}_{\mu}^{E}(D, \mathcal{S})$ is connected.

Proof. If not, we have compact disjoint non-empty sets whose union is $\mathcal{T}_{\mu}^{E}(D, \mathcal{S})$. The minimum distance is obtained, say, by $\mathcal{S}$-fibers $K_{1}$ and $K_{2}$. Then $K_{1} \vee K_{2}$ and $K_{1} \wedge K_{2}$, and every fiber in an $\mathcal{S}$-pseudo-roof of $D\left(K_{1} \vee K_{2}, K_{1} \wedge K_{2}\right)$, are all in $\mathcal{T}_{\mu}^{E}(D, \mathcal{S})$. Such a roof will contain a $\mu$-fiber, and any fiber $L \in D\left(K_{1} \vee K_{2}, K_{1} \wedge K_{2}\right)$ satisfies $d_{\mathcal{S}}\left(K_{1}, L\right)<d_{\mathcal{S}}\left(K_{1}, K_{2}\right)$. This is a contradiction.

Lemma 8.11. If $(D, \mathcal{S})$ is a directed pseudo-domain of order $\operatorname{ord}(\mathcal{S})=2 n$, then every connected component of the compact space $\mathcal{V} h_{\mu}^{(2 n-2)}(D)$ containing a central $\mu$-fiber contains an $\mathcal{S}$-central $\mu$-fiber. 
Proof. Let $F$ be a central $\mu$-fiber of $\mathcal{V} h_{\mu}^{(2 n-2)}(D)$, and let $F_{1}$ and $F_{2}$ be as in Definition 6.18. If $F$ is an $\mathcal{S}$-fiber there is nothing to prove, so we may assume that $F_{1}<F<F_{2}$. There will be an even number of rectangles from $\mathcal{S}$ meeting the rectangle determined by $F_{1}$ and $F_{2}$, and if $E$ denotes the set of east-west borders of these rectangles, then $|E| \geq 1$ and there is an $\mathcal{S}$-pseudo-roof of $D(F, F)$ all of whose fibers are in $\mathcal{T}_{\mu}^{E}(D, \mathcal{S})$. Hence we have an $\mathcal{S}$-central $\mu$-fiber $G$ with $\operatorname{ord}(G) \leq 2 n-2$ and $F_{1}<G<F_{2}$. By Lemma 8.7, $F$ and $G$ are in the same connected component of $\mathcal{V} h_{\mu}^{(2 n-2)}(D)$.

By combining these lemmas with Lemma 6.38(iv), we get another key result.

Proposition 8.12. If $(D, \mathcal{S})$ is an eligible directed pseudo-domain with $\operatorname{ord}(\mathcal{S})=2 n$ and $\mu(D)=1$, then the compact space $\mathcal{V} h_{\mu}^{(2 n-2)}(D)$ is nonempty and connected.

Proof. Note that in the exceptional case $M<L$ in Lemma 6.38(iv), it follows from Lemma 8.2 that $\mathcal{V} h^{(2 n-2)}(D(M, L))=\mathcal{V} h_{\mu}^{(2 n)}(D(M, L))$, and $\mathcal{V} h^{(2 n-2)}(D(M, L))$ is connected since any fiber in $D(M, L)$ of order not exceeding $2 n-2$ can occur in a pseudo-roof for $D(M, L)$.

9. Lifting $\mathbf{v h}_{\sigma}$-fibers right. We fix notation as in the previous sections, and add to this the following special fiber, and its lifts to $Z$.

Definition 9.1. We denote by $I$ the fiber whose curve $\gamma$ is given by $\widetilde{x}_{1}(\gamma(t))=t(\bmod 1)$ and $\widetilde{x}_{2}(\gamma(t))=0(\bmod 1)$. The lift of $I$ to $Z$, whose curve $\widetilde{\gamma}^{(n)}$ is given by $\widetilde{x}_{1}\left(\gamma^{(n)}(t)\right)=t(\bmod 1)$ and $x_{2}\left(\gamma^{(n)}(t)\right)=n$, is denoted by $\widetilde{I}^{(n)}$. We call $I$ the equator.

Without loss of generality, we assume that $\mu(I)=1$. Note that $\widetilde{\mathcal{V} h}(0)=$ $\left\{\widetilde{I}^{n} \mid n \in \mathbb{Z}\right\}$ is just a discrete space. We next look into the connectivity of $\widetilde{\mathcal{V} h}(2 n)$ for all $n \in \mathbb{N}$.

DEFINITION 9.2. We denote by $\widetilde{\mathcal{W} h}(2 n)$ the subspace of $\widetilde{\mathcal{V} h} h_{\mu}^{(2 n)} \times \widetilde{\mathcal{V} h}(2 n-2)$ consisting of pairs $\left(\widetilde{K}, \widetilde{K}^{\prime}\right)$ such that $\widetilde{K}$ and $\widetilde{K}$ are lifts of $\mu$-fibers, and $\widetilde{K}^{\prime} \in \widetilde{\mathcal{V} h}{ }_{\mu}^{(2 n-2)}(\widetilde{D}(\widetilde{K}))$. We let $p_{1}$ and $p_{2}$ be the two projections from $\widetilde{\mathcal{W}}_{\mu}^{(2 n)}$. If $\phi:[a, b] \rightarrow \widetilde{\mathcal{V} h}{ }_{\mu}^{(2 n)}$ is a continuous map, we simply denote by $\widetilde{\mathcal{W} h}(\phi)$ the pullback of the image of $\phi$ by $p_{1}$.

Definition 9.3. Let $\phi:[a, b] \rightarrow \widetilde{\mathcal{V} h}(2 n)$ be a continuous map, and suppose $\operatorname{ord}(\phi(t))=2 n$ for all $t$. We can choose a matching $w$. For any set of $E \subseteq \operatorname{circocc}(x x, w)$, we let $\widetilde{\mathcal{W} h}{ }_{E}(\phi)$ denote the subspace of fibers $K^{\prime} \in \widetilde{\mathcal{V} h}_{\mu}^{(2 n-2)}\left(\widetilde{D}_{E}(\phi(t))\right)$. 
LEMMA 9.4. Let $\phi:[a, b] \rightarrow \widetilde{\mathcal{V} h}(2 n)$ be a continuous map, and suppose $\operatorname{ord}(\phi(t))=2 n$ for all $t \in\langle a, b\rangle$, and $\operatorname{ord}(\phi(t))<2 n$ for $t \in\{a, b\}$. Then $\widehat{\mathcal{W h}}(\phi)$ is connected, and $\{\phi(a), \phi(b)\}$ belong to the same component of $\widetilde{\mathcal{V} h}(2 n-2)$.

Proof. Connectedness of $\widetilde{\mathcal{W} h}(\phi)$ follows from Proposition 8.12, Lemma 8.1, and Corollary 7.6, and the last statement follows from Proposition 7.7.

COROLlaRY 9.5. Inclusion induces a one-to-one correspondence between the connected components of $\widetilde{\mathcal{V} h}(2 n-2)$ and $\widetilde{\mathcal{V} h}(2 n)$.

COROLlaRY 9.6. There is a unique surjective function, which we call $R$, from $\widetilde{\mathcal{V h}}_{\mu}$ to $\mathbb{Z}$ with the property that that it is continuous on each subspace $\widetilde{\mathcal{V h}}_{\mu}^{(2 n)}$, and takes the value $n$ at the fiber $\widetilde{I}^{(n)}$.

Proof. By induction on $n$, every $\mu$-fiber of order $2 n$ belongs to a connected component of $\widetilde{\mathcal{V} h}(2 n)$ that contains exactly one of the fibers $\widetilde{I}^{(n)}$.

Let $B$ be the set of $\operatorname{vh}_{\sigma}$-domains $\widetilde{D}=\widetilde{D}\left(\widetilde{K}, \widetilde{K}^{\prime}\right)$ such that $\widetilde{K} \cap \widetilde{K}^{\prime}=\emptyset$, $\mu(K)=\mu\left(K^{\prime}\right)=1$ and $R(\widetilde{K})=R\left(\widetilde{K}^{\prime}\right)=0$. The set $B$ is graded by the function $\operatorname{ord}(\widetilde{D})=\operatorname{ord}(\widetilde{K})+\operatorname{ord}\left(\widetilde{K}^{\prime}\right)$. We will eventually see that $B=\emptyset$, but first we prove some properties of domains in $B$ if there are any. Recall the concepts of snug obstructions and $(\varepsilon, \delta)$-immobility of Definitions 5.18 and 5.15 .

Lemma 9.7. If $B \neq \emptyset$, it contains snugly reduced $(\varepsilon, \delta)$-immobile domains for some $\varepsilon>\delta>0$.

Proof. Let $\widetilde{D}\left(\widetilde{K}, \widetilde{K}^{\prime}\right)$ be a member of $B$. If $K$ has a $\left\{K, K^{\prime}\right\}$-unobstructed left tag or stair-like turn, the pseudo-domain obtained by adding the closure of its shadow and the closures of the shadows of all $\{K\}$-unobstructed right tags, which are automatically also $\left\{K^{\prime}\right\}$-unobstructed, has right and left boundaries of order strictly smaller than ord $(K)$, and building a pseudo-roof over it gives us a fiber $K_{1}$ with a lift $\widetilde{K}_{1}$ such that $\widetilde{D}\left(\widetilde{K}_{1}, \widetilde{K}^{\prime}\right)$ is also a member of $B$ with strictly smaller order. We may therefore assume that $\widetilde{D}\left(\widetilde{K}, \widetilde{K}^{\prime}\right)$ is reduced. If $\widetilde{D}\left(\widetilde{K}, \widetilde{K}^{\prime}\right)$ is reduced, but not necessarily snugly so, consider the pseudo-domain $D$ obtained by adding closed rectangles on the left side of $K$ to make every $\left\{K^{\prime}\right\}$-primary-obstructed left turn of the left boundary of $D$ snugly $\left\{K^{\prime}\right\}$-obstructed. Then add the closures of the shadows of all $\{K\}$-unobstructed right tags. We choose a reducing pseudo-roof such that there is a middle fiber to the left of all the rectangles added on the left side of $K$ and to the right of all the rectangles added on the right side of $K$. If there is a $\mu$-fiber on the left side of the middle fiber, this fiber, say $K_{1}$, will have all its $\left\{K^{\prime}\right\}$-primary-obstructed left turns snugly $\left\{K^{\prime}\right\}$-obstructed. If not we 
repeat the process. The order of the left side of each new domain will decrease each time, so eventually we end up with a domain $\widetilde{D}\left(\widetilde{K}, \widetilde{K}^{\prime}\right)$ such that all $\left\{K^{\prime}\right\}$-primary-obstructed left turns of $K$ are snugly $\left\{K^{\prime}\right\}$-obstructed. We can then switch the roles of $K$ and $K^{\prime}$ and obtain a domain $\widetilde{D}\left(\widetilde{K}, \widetilde{K}^{\prime}\right)$ such that all $\{K\}$-primary-obstructed right turns of $K^{\prime}$ are snugly $\{K\}$-obstructed. In the process, however, some $\left\{K^{\prime}\right\}$-primary-obstructed left turns of $K$ may become $\left\{K^{\prime}\right\}$-unobstructed, but then we may reduce the order and simply cut off the shadow of that turn. Note that a snugly obstructed turn does not become non-snugly obstructed. Alternating this process between $K$ and $K^{\prime}$ always decreases the order so eventually we end up with a snugly reduced domain. Let $0<\delta<\varepsilon$ and $n$ be such that there is a snugly reduced domain $\widetilde{D}\left(\widetilde{K}, \widetilde{K}^{\prime}\right)$ of $B$ with the $(\varepsilon, \delta)$-property and with $\operatorname{ord}(D)=n$. Let $S B_{(\varepsilon, \delta)}^{n}$ be the subset of $B$ consisting of the snugly reduced domain $\widetilde{D}\left(\widetilde{K}, \widetilde{K}^{\prime}\right)$ of $B$ with the $(\varepsilon, \delta)$-property and with $\operatorname{ord}(D) \leq n$. We have just shown that there exist $0<\delta<\varepsilon$ and $n$ such that $S B_{(\varepsilon, \delta)}^{n} \neq \emptyset$. The space $S B_{(\varepsilon, \delta)}^{n}$ is compact because of Definition 0.1(b) and the continuity of distance. Since the area of a domain $\widetilde{D}=\widetilde{D}\left(\widetilde{K}, \widetilde{K}^{\prime}\right)$ is continuous on $B$, there is a domain $\widetilde{D}\left(\widetilde{K}, \widetilde{K}^{\prime}\right)$ in $S B_{(\varepsilon, \delta)}^{n}$ of minimum area. We claim that $\widetilde{D}\left(\widetilde{K}, \widetilde{K}^{\prime}\right)$ is $(\varepsilon, \delta)$-immobile. If $K$ had a left movable leg, we could use an unobstructed right tag to move it to the left and possibly gain some area because the right tag might shrink too much. However, alternating the process back and forth between $K$ and $K^{\prime}$ eventually produces a snugly reduced pair of smaller area in the same connected component.

We can now formulate our final lemma.

Lemma 9.8. If $\widetilde{K}$ and $\widetilde{K}^{\prime}$ are lifts of $\mu$-fibers $K$ and $K^{\prime}$ such that $R(\widetilde{K})=R\left(\widetilde{K}^{\prime}\right)$, then $\widetilde{K} \cap \widetilde{K}^{\prime} \neq \emptyset$.

Proof. It follows from Lemmas 9.7 and 5.25 that $B=\emptyset$, which is a reformulation of the lemma.

10. Density and connectedness. As before, let $p: Y \rightarrow X$ be a universal covering of a torus, and let $(x, y): Y \rightarrow \mathbb{R}^{2}$ be coordinates such that the unit square is a fundamental domain for $p$. Let $\mathbb{P}$ be the set of maximal rank one subgroups of the fundamental group $\pi_{1}(X)$. For each $\sigma \in \mathbb{P}$, and each simple topological measure $\varrho \in X^{*}$, let $\varrho_{\sigma}$ be the restriction of $\varrho$ to the $\sigma$-sets. We let $X_{\sigma}^{*}$ denote the corresponding quotient space, with the quotient topology. Then if we give $\prod_{\sigma \in \mathbb{P}} X_{\sigma}^{*}$ the product topology and $X_{\text {int }}^{*}$ the subspace topology, the natural map

$$
X_{\mathrm{int}}^{*} \rightarrow \prod_{\sigma \in \mathbb{P}} X_{\sigma}^{*}
$$


is a homeomorphism. According to Ørjan Johansen and Alf Birger Rustad the map $\lambda: X^{*} \rightarrow X_{\text {int }}^{*}$ is a deformation retraction so the inclusion $X_{\text {int }}^{*} \subseteq X^{*}$ of the intrinsic simple topological measures into all the simple topological measures induces isomorphisms on the Cech cohomology groups (see [8]). It follows that $X^{*}$ is connected if and only if $X_{\mathrm{int}}^{*}$ is connected.

DeFinition 10.1. Let $D_{n} \subseteq Y$ be the square given by $-n \leq x \leq n$ and $-n \leq y \leq n$, and let $i_{n}$ denote the inclusion. We set $X_{n}^{*}=\lambda \circ\left(p \circ i_{n}\right)_{*}\left(D_{n}^{*}\right)$.

Proposition 10.2. The union $\bigcup_{n=1}^{\infty} X_{n}^{*}$ is dense in $X_{\text {int }}^{*}$.

Proof. Let $\varrho$ be an intrinsic simple topological measure and let $\mathcal{F}$ be a finite family of two-sided open sets with $\varrho$-measure 1 . Let $U$ be the neighborhood of $\varrho$ given by $\{\delta \mid \delta(V)=1, \forall V \in \mathcal{F}\}$. By Theorem 1.9, there is a lifting $\mathcal{F}^{\prime}$ of $\mathcal{F}$ to $Y$ (see Definition 1.2). For each $V^{\prime} \in \mathcal{F}^{\prime}$ choose a point $p_{V^{\prime}} \in V^{\prime}$, and for each pair $\left(V^{\prime}, W^{\prime}\right)$ with $V^{\prime} \neq W^{\prime}$ a point $q_{\left(V^{\prime}, W^{\prime}\right)} \in V^{\prime} \cap W^{\prime}$. Choose curves $\gamma_{\left(V^{\prime}, W^{\prime}\right)}$ from $p_{V^{\prime}}$ to $q_{\left(V^{\prime}, W^{\prime}\right)}$ in $V^{\prime}$, and let $C_{V^{\prime}}=\bigcup_{W^{\prime} \neq V^{\prime}} \gamma_{\left(V^{\prime}, W^{\prime}\right)}$. Then $C_{V^{\prime}}$ is compact. For each $V^{\prime} \in \mathcal{F}^{\prime}$, we have $C_{V^{\prime}} \subseteq V^{\prime}$, and the family $\left\{C_{V^{\prime}} \mid V^{\prime} \in \mathcal{F}^{\prime}\right\}$ is strongly linked. By compactness there is an $n$ large enough so that all the curves $\gamma_{\left(V^{\prime}, W^{\prime}\right)}$ lie in $D_{n}$. It follows that the family $\mathcal{F}^{\prime} \cap D_{n}=\left\{V^{\prime} \cap D_{n} \mid V^{\prime} \in \mathcal{F}^{\prime}\right\}$ is strongly linked so there is a generalized point-measure $\delta \in D_{n}^{*}$ with $\delta\left(V^{\prime} \cap D_{n}\right)=1$ for all $V^{\prime} \in \mathcal{F}^{\prime}$, which shows that $X_{n}^{*} \cap U \neq \emptyset$.

Since the maps $\lambda \circ\left(p \circ i_{n}\right)_{*}$ are continuous it follows that $X_{n}^{*}$ is a connected subset of $X_{\text {int }}^{*}$. Also, $X_{n}^{*} \subseteq X_{n+1}^{*}$ for each $n$. It follows that $\bigcup_{n=1}^{\infty} X_{n}^{*}$ is connected, and hence we have the corollary:

Corollary 10.3. The spaces $X_{\mathrm{int}}^{*}$ and $X^{*}$ are connected.

Acknowledgments. I am particularly grateful to the referee, whose suggestions significantly improved the readability of the article.

\section{References}

[1] J. Aarnes, Quasi-states and quasi-measures, Adv. Math. 86 (1991), 41-67.

[2] - Construction of non-subadditive measures and discretization of Borel measures, Fund. Math. 147 (1995), 213-237.

[3] S. V. Butler, Density of the space of topological measures, ibid. 174 (2002), 239-251.

[4] - Density of the space of extreme topological measures, ibid. 192 (2006), 141-153.

[5] M. Entov and L. Polterovich, Quasi-states and symplectic intersections, Comment. Math. Helv. 81 (2006), 75-99.

[6] D. J. Grubb, Irreducible partitions and the construction of quasi-measures, Trans. Amer. Math. Soc. 353 (2001), 2059-2072.

[7] D. J. Grubb and T. LaBerge, Spaces of quasi-measures, Canad. Math. Bull. 42 (1999), 291-297. 
[8] Ø. Johansen and A. B. Rustad, The homology of spaces of simple topological measures, Fund. Math. 177 (2003), 19-43.

[9] F. F. Knudsen, Topology and the construction of extreme quasi-measures, Adv. Math. 120 (1996), 302-321.

[10] - New topological measures on the torus, Fund. Math. 185 (2005), 287-293.

[11] T. LaBerge, Supports of quasi-measures, Houston J. Math. 24 (1998), 301-312.

Department of Mathematical Sciences

Norwegian University of Science and Technology

NO-7491 Trondheim, Norway

E-mail: knudsen@math.ntnu.no

Received 4 December 2006;

in revised form 6 May 2008 